\title{
New Extreme Trans-Neptunian Objects: Towards a Super-Earth in the Outer Solar System
}

\author{
Scott S. Sheppard ${ }^{1}$ and Chadwick Trujillo ${ }^{2}$
}

\begin{abstract}
We are conducting a wide and deep survey for extreme distant solar system objects. Our goal is to understand the high perihelion objects Sedna and 2012 VP113 and determine if an unknown massive planet exists in the outer solar system. The discovery of new extreme objects from our survey of some 1080 square degrees of sky to over 24th magnitude in the $r$-band are reported. Two of the new objects, 2014 SR349 and 2013 FT28, are extreme detached trans-Neptunian objects, which have semi-major axes greater than $150 \mathrm{AU}$ and perihelia well beyond Neptune $(q>40 \mathrm{AU})$. Both new objects have orbits with arguments of perihelia within the range of the clustering of this angle seen in the other known extreme objects. One of these objects, 2014 SR349, has a longitude of perihelion similar to the other extreme objects, but 2013 FT28 is about 180 degrees away or anti-aligned in its longitude of perihelion. We also discovered the first outer Oort cloud object with a perihelion beyond Neptune, 2014 FE72. We discuss these and other interesting objects discovered in our ongoing survey. All the high semi-major axis $(a>150 \mathrm{AU})$ and high perihelion $(q>35 \mathrm{AU})$ bodies follow the previously identified argument of perihelion clustering as first reported and explained as being from an unknown massive planet by Trujillo and Sheppard (2014), which some have called Planet X or Planet 9. With the discovery of 2013 FT28 on the opposite side of the sky, we now report that the argument of perihelion is significantly correlated with the longitude of perihelion and orbit pole angles for extreme objects and find there are two distinct extreme clusterings anti-aligned with each other. This previously unnoticed correlation is further evidence of an unknown massive planet on a distant eccentric inclined orbit, as extreme eccentric objects with perihelia on opposite sides of the sky (180 degree longitude of perihelion differences) would approach the inclined planet at opposite points in their orbits, thus making the extreme objects prefer to stay away
\end{abstract}

\footnotetext{
${ }^{1}$ Department of Terrestrial Magnetism, Carnegie Institution for Science, 5241 Broad Branch Rd. NW, Washington, DC 20015, USA, ssheppard@carnegiescience.edu

${ }^{2}$ Gemini Observatory, 670 North A'ohoku Place, Hilo, HI 96720, USA
} 
from opposite ecliptic latitudes to avoid the planet (i.e. opposite argument of perihelia or orbit pole angles).

Subject headings: Kuiper belt: general - Oort Cloud - comets: general - minor planets, asteroids: general - planets and satellites: individual (Sedna, 2012 VP113, 2014 SS349, 2014 SR349, 2013 FT28, 2014 FE72)

\section{Introduction}

Extreme trans-Neptunian objects (ETNOs) have orbits that are not easily explained through the currently observed solar system configuration. Objects like Sedna and 2012 VP113 are the two most prominent members of the ETNOs (Trujillo and Sheppard 2014). Their large perihelia of 76 and $80 \mathrm{AU}$ respectively, mean they are decoupled from the giant planet region, but yet have highly eccentric orbits (Figures 1 and 2). These objects must have interacted with something in the past to obtain their distant, eccentric orbits. Unlike objects in the outer Oort cloud $(a>1500 \mathrm{AU})$, which can have their orbits modified by interactions with the galactic tide, Sedna and 2012 VP113 are too tightly bound to the Sun to be strongly perturbed by outside forces. For this reason, Sedna and 2012 VP113 have been called inner Oort cloud (IOC) objects.

Several viable theories have been put forth to explain the orbits of IOC objects (Kenyon and Bromley 2004; Morbidelli and Levison 2004; Brown et al. 2004; Melita et al. 2005; Levison et al. 2010; Kaib et al. 2011; Jilkova et al. 2015; Portegies Zwart and Jilkova 2015). The most popular scenario, until recently, is that the IOC objects were put into place while the Sun was still within its birth cluster (Brasser et al. 2012). During this time, many other young forming stars were near the Sun and thus outside forces, such as the stellar tide from nearby stars, were much stronger than today. The stellar tide from the Sun's birth cluster could have perturbed objects much closer to the Sun, possibly down to hundreds of AU if the Sun formed in a very dense stellar environment (Brasser et al. 2006). In this way, IOC objects would be a fossilized population of objects with major changes to their orbits ending when our Sun left the star formation cluster. This would suggest studying the IOC objects would allow us to gain insights into our Sun's formation environment (Schwamb et al. 2010).

The IOC objects could also have been created by rogue planets that were scattered or ejected from the solar system (Morbidelli and Levison 2004; Brown et al. 2004; Gladman and Chan 2006). The involvement of planetary perturbers in the IOC formation gained additional traction with the observation that Sedna and 2012 VP113, along with ten other extreme trans-Neptunian objects $(q>35 \mathrm{AU}$ and $a>150 \mathrm{AU})$, have similarities in their 
orbits (Trujillo and Sheppard 2014). These twelve extreme objects cluster within a few tens of degrees of 340 degrees in their argument of perihelia and are near the same location on the sky. Trujillo and Sheppard (2014) demonstrated this unexpected orbital clustering could be created by an unseen planet some 2 to 15 Earth masses beyond a few hundred AU, which is currently shepherding these distant objects into similar orbits likely through some sort of resonance dynamics.

With the idea that there is an unseen massive planet in the few hundred AU range from Trujillo and Sheppard (2014), Bromley and Kenyon (2014) and Kenyon and Bromley (2015) showed how a massive planet might have obtained such a distant orbit after being scattered out of the giant planet region and interacting with leftover gas from the planet formation era. It also seems plausible that the stellar tide formation originally envisioned for Sedna would also work for the formation of the unseen planet's distant orbit. Thus the unseen planet may have begun forming near the known giant planets but was placed into a distant orbit before it accreted a larger amount of mass. Because of the quite different semi-major axes of the ETNOs, de la Fuente Marcos and de la Fuente Marcos (2014a) suggested two planets instead of just one might be responsible for the clustering of ETNOs. Several authors further showed how this distant massive planet might influence other bodies in the solar system such as the extreme Centaurs (de la Fuente Marcos and de la Fuente Marcos 2014b; Gomes et al. 2015). Hees et al. (2014) and Iorio (2014) tried to put limits on the size and distance of the possible unseen massive planet through looking at the Cassini spacecraft tracking data and planetary ephemerides for unexplained gravitational perturbations.

The work by Trujillo and Sheppard (2014) (From here on called TS2014) identified the surprising result that the ETNOs clustered in their orbital parameters. They demonstrated that this clustering was not from observational biases as most of the ETNOs were discovered at high ecliptic latitudes. Further, it was shown the clustering of ETNOs would only be seen if the mechanism for the clustering of the ETNOs is still operating, as any orbital clustering from past situations would disperse over time if no continuing shepherding mechanism is in place. TS2014 then showed the basic proof of concept that a yet unobserved outer massive planet beyond a few hundred astronomical units was likely responsible and could create the ETNO clustering. TS2014 did some basic numerical simulations to show that a planet would need to be larger than about 2 Earth masses to shepherd the orbital angles of these distant extreme trans-Neptunian objects and that this planet would need to be beyond about 200 AU to have gone undetected to date. Although TS2014 presented a 5 Earth mass planet at $250 \mathrm{AU}$ in their simulation figure, TS2014 specifically mentioned that there were many possibilities for the orbit of the unknown massive planet and never specified a best or unique orbit as much more parameter space needed to be explored then in their initial simulations. 
The main parameters identified by TS2014 for the distant outer planet were a mass between 2 and 15 Earth masses with a distance beyond 200 AU, with a highly inclined 1500 AU orbit being possible. TS2014 suggested that a more comprehensive, wider orbital parameter analysis would put tighter constraints on the possible orbits for a unknown outer planet. Two years later, Batygin and Brown (2016) (hereafter BB2016) took this next step with a more rigorous analytic treatment coupled with dynamical simulations inspired by the ETNO clustering suggested by TS2014. BB2016 confirmed that there is indeed a clustering of ETNOs in argument of perihelion and longitude of perihelion. They placed stronger constraints on the possible orbit of the massive unknown planet, showing that the pertuber is likely on an eccentric orbit that is inclined and anti-aligned with the ETNOs with a perihelion no closer than about $200 \mathrm{AU}$ and aphelion no further than about $1500 \mathrm{AU}$. The BB2016 result further predicts the highly inclined and retrograde TNO population (see Gladman et al. 2009) is also created by the unseen planet. Malhotra et al. (2016) further looked into the clustering of the ETNO population and suggest that some of the ETNOs have orbital period ratios indicative of being in mean motion resonances with an unknown distant planet.

Some authors have called this possible massive planet beyond a few hundred astronomical units Planet X or Planet 9. Fienga et al. (2016) and Holman \& Payne (2016a,b) have looked further into the Cassini tracking and planetary ephemerides to put limits on the size and distance of an unseen massive planet in the outer solar system. Sheppard et al. (2016) showed that this distant planet likely has no significant effect on the orbits of objects with semi-major axes within $100 \mathrm{AU}$ and moderate or lower eccentricities, including objects in the distant 6:1 Neptune mean motion resonance near 99 AU. Scattered objects with very distant orbits will likely start to show effects from the distant planet (Lawler et al. 2016). Several additional papers have discussed the possible formation, effects and composition of the unseen planet in the distant solar system (Kenyon and Bromley 2016; Fortney et al. 2016; Sivaram et al. 2016; de la Fuente Marcos and de la Fuente Marcos 2016; Li and Adams 2016; Cowan et al. 2016; Beust 2016; Linder and Mordasini 2016; Pauco and Klacka 2016; Mustill et al. 2016; Brown and Firth 2016; Whitmire 2016; Gomes et al. 2016).

We note Madigan and McCourt (2016) recently showed that eccentric small objects within a massive disk would increase their inclinations and could clump into similar arguments of perihelia from a gravitational instability between the bodies and the disk. Though intriguing, it is not clear these objects would continue to cluster once the massive disk is eroded away as quadrupolar interactions with the inner giant planets would randomize the arguments of perihelia once again as discussed in TS2014. Future work is needed, especially to see if it might be possible that the mass from the more distant outer Oort cloud could have some dynamical effect on the Inner Oort Cloud objects. 
IOC objects and ETNOs have the potential to inform us about our solar system's origins and evolution, but yet the number of ETNOs is currently small. For this reason, we have been performing the deepest, widest survey to date for objects beyond the Kuiper Belt edge at $50 \mathrm{AU}$. Here we report several more interesting objects found in the distant solar system and discuss what new information they might be telling us about the outer solar system. This is an ongoing survey, so this paper highlights the first years of coverage and the interesting discoveries that have been made. The discovery and implications of the inner Oort cloud object 2012 VP113 was reported in TS2014 and so is only discussed tangentially in this paper. In addition, several high perihelion objects $(q>50 \mathrm{AU})$ with only moderate semimajor axes $(50<a<100 \mathrm{AU})$ and eccentricities $(e \lesssim 0.3)$ were found, such as $2014 \mathrm{FZ71}$ and 2015 FJ345, and reported in Sheppard et al. (2016). These high perihelion but moderate orbit objects are all near strong Neptune Mean Motion Resonances with moderate to high inclinations. These objects likely obtained their unusual high perihelion orbits through interactions with the Neptune mean motion resonances and the Kozai resonance and thus likely have a different history than the extreme objects reported in this paper.

If there is a distant massive planet shepherding the extreme trans-Neptunian objects, it implies that the IOC population is not in fact primordial as their orbits would be modified by the unseen planet and not static and related to the formation of the Oort cloud as originally thought. Thus, a different nomenclature than IOC, such as sednoids, would be best to use when discussing bodies like Sedna and 2012 VP113. Since the possible unseen planet has not been discovered and no such nomenclature has been agreed upon, in this work we will use IOC to refer to bodies with extremely high perihelia $(q>70 \mathrm{AU})$ for consistency with past works.

\section{Observations}

The majority of the area surveyed was with the Cerro Tololo Inter-American Observatory (CTIO) 4 meter Blanco telescope in Chile with the 2.7 square degree Dark Energy Camera (DECam). DECam has $622048 \times 4096$ pixel CCD chips from Lawrence Livermore Berkeley Labs with a scale of 0.26 arcseconds per pixel (Flaugher et al. 2015). The r-band filter was used during the early observing runs (November and December 2012 and March, May and November 2013) and the ultra-wide $V R$ filter was used in the later observations (March and September 2014 and April 2015).

Before DECam became operational the initial inner Oort cloud survey was begun using the 0.255 square degree SuprimeCam on the 8 meter Subaru telescope (Miyazaki et al. 2002),

the 0.16 sq. deg. IMACS on the 6.5 meter Magellan telescope (Dressler et al. 2011) and the 
0.36 sq. deg. Mosaic-1.1 on the Kitt Peak National Observatory (KPNO) 4 meter Mayall telescope (Jacoby et al. 1998; Wolfe et al. 1998; Sawyer et al. 2010). The observing nights and conditions of the survey fields are shown in Table 1 and Figure 3.

Usable survey data required no significant extinction from clouds and seeing less than 1.5 arcseconds at the $\mathrm{CTIO} 4 \mathrm{~m}$ and $\mathrm{KPNO} 4 \mathrm{~m}$. In general the exposure times were set to reach 24th magnitude with the r-band filter and 24.5 magnitude with the VR filter during the night. In the best seeing of 0.8 arcseconds, integration times were around 330 seconds, while in the worst seeing exposure times were up to 700 seconds. This allowed our survey to obtain a similar depth regardless of the seeing conditions. The Subaru and Magellan observations went deeper, with the target depth of around 25.5 magnitudes in the r-band and useful seeing being less than 1.0 arcseconds.

The images were all processed in a similar manner. Images were first bias-subtracted and then flat fielded with a nightly combination of twilight flats and master flats created from most of the data in a single night. The telescope was autoguided sidereally on field stars during exposures. Most fields had three images of similar depth obtained over a 3 to 5 hour time base (Table 1). Because of observing condition changes and night length constraints, a few images had a slightly smaller time base while others had up to 48 hours between the first and last image. Observations were obtained within about 1.5 hours of opposition, which means the dominant apparent motion of any distant object would be parallactic, and thus inversely related to distance.

We used a moving object algorithm to detect objects moving at rates indicative of being beyond Uranus (less than 5 arcseconds per hour). We were in general sensitive to objects that were about 3 sigma above the background and moved at least 3 to 4 pixels between the first and last image of a 3 image set. The program has been extensively used in discoveries of distant solar system objects (Trujillo et al. 2001; Sheppard and Trujillo 2006,2009,2010a) and was slightly modified from previous versions, such as the pixel scale, field orientations and saturation limits, to operate on the different wide-field optical instruments used in this survey (see Trujillo and Sheppard 2014). The program first determined all possible objects moving at the desired rates. One of the authors then visually inspected all images with the moving objects reported by the program circled to determine which objects were real and which were noise from things such as cosmic rays, saturated stars or bad pixels. As the eye is very good at picking out real objects from noise, it was straight forward to determine which objects to obtain additional observations. Any discovery beyond 50 AU was flagged for future recovery observations to determine if it had an extreme orbit.

Through implanting false objects into our fields, we found most fields $(\sim 90 \%)$ we were sensitive to objects moving faster than about 0.3 arcseconds per hour, which corresponds 
to about $500 \mathrm{AU}$ at opposition. About $25 \%$ of the sky covered in this survey could detect motions to $700 \mathrm{AU}$ or beyond (generally fields that have over a 4 hour time-base, see Table 1). Some $14 \%$ of the fields had about a 24 hour time-base between the first and last images, allowing objects out to a few thousand AU to be detected. False objects of varying speeds and magnitudes were implanted into a few representative fields of an observing run and put through the program and visually scanned just like the rest of the fields to determine the efficiency and limits of the observing session.

We generally stayed some 15 degrees away from the galactic plane as shown in Figure 3 . There are a few fields within the galactic plane that used known dark clouds to search areas with low stellar background as described in Sheppard and Trujillo 2010b. Most of the survey fields were between 5 and 20 degrees from the ecliptic, with an average of about 13 degrees from the ecliptic. The fields have a fairly uniform longitudinal coverage and are mostly in the southern sky (Figure 3). This work is about the first 1080 square degrees covered to date to over 24th magnitude (Subaru=40, Magellan=60, KPNO=11 and DECam $=970$ square degrees).

\section{Observational Bias Simulator For Population and Orbit Statistics}

We created an observational bias simulator to place population estimates and compute orbital statistics on the extreme and the inner Oort cloud objects. For our input, we have taken the positions and depths of fields listed in Table 1. Since mean anomalies were randomized for all simulated populations, ephemerides were computed for only a single date to speed execution time. A simulated object was considered to be detected when located in one of our survey fields with apparent brightness greater than our survey limit. For detection, an object also had to have heliocentric distance greater than our survey lower limit for followup, $50 \mathrm{AU}$, and distance less than $500 \mathrm{AU}$, where opposition apparent motion falls below our $\sim 0.28$ arcsec per hour detection limit for many of our fields. We have not included data from other surveys in this work mainly because their field locations and depths are not known to us and they typically used smaller observation time-base and smaller telescopes and were therefore less sensitive to very distant objects.

We had several basic input parameters that were used for all of our populations. Since very few numbers of IOCs and ETNOs are known, our size distribution is based on the hot component of the TNOs, which have a luminosity function slope of $\alpha_{h}=0.8$ (Petit et al. 2011; Schwamb et al. 2014). This is the equivalent of a differential power law to the size distribution of $q^{\prime}=-5$ (Trujillo 2000). Both the IOCs and ETNOs could share

origins with the hot component of the TNOs since none of these populations is thought to 
have formed in their current locations (Tsiganis et al 2005). As shown in section 5.4 below, the IOCs and ETNOs appear to have a similar cumulative luminosity function slope as the

other small body populations and thus likely follow the same $q^{\prime} \sim-5$ size distribution. For completeness, we also simulated a size distribution of $q^{\prime}=-4$.

As mentioned in section 4 below, we find the average inclination of the ETNOs to be similar as that found for the scattered disk objects by Gulbis et al. (2010). For this reason the inclination distribution is assumed to be similar to the scattered TNOs with an average around $\mu=19.1 \mathrm{deg}$ and a gaussian of $\sigma=6.9 \mathrm{deg}$ (Gulbis et al. 2010). Other simulationwide parameters are largely assumed since there is very little observational constraint on them and are detailed later.

\section{Results: New Extreme Trans-Neptunian Objects}

This survey has now discovered new ETNOs that could have an origin similar to the IOC objects and thus could constrain observations of dynamical processes in the outer solar system beyond the Kuiper Belt. The orbital elements of the newly discovered objects plotted with the well known outer solar system objects are shown in Figures 4 to 10. The figures are divided between objects with perihelia greater than and less than 35 AU. This perihelion cutoff value might be better chosen even great than 35 AU as current Neptune interactions are likely significant up to at least 38-40 AU (Gomes et al. 2008) or 41 AU (Brasser and Schwamb 2015) for objects with semi-major axes greater than 250 AU. We use 35 AU simply because from an observational perspective, this is where the orbital data for extreme objects appears to start to cluster for the argument of perihelion. We note that since the extreme objects start to cluster at a perihelion of $35 \mathrm{AU}$, where Neptune interactions are still fairly strong, this suggests the mechanism that is creating the clustering is stronger than the Neptune interactions beyond a perihelion of $35 \mathrm{AU}$.

The ETNOs other than Sedna and 2012 VP113 have perihelia less than 50 AU and thus are not as decoupled from the giant planet region as the IOC objects and thus their origins could be similar to those of Sedna and 2012 VP113 or they could have more significant modifications from Neptune interactions. It appears the few objects with perihelia above 40 AU but below 50 AU with high eccentricities are likely to have a similar origin as Sedna and 2012 VP113 as their interactions with Neptune should be minimal (Gladman et al. 2002; Gomes et al. 2008; Chen et al. 2013; Brasser and Schwamb 2015). In this work, we call objects with $40<q<50 \mathrm{AU}$ and semi-major axes greater than 150 and less than $1500 \mathrm{AU}$ extreme detached trans-Neptunian objects (EDTNOs). These extreme detached objects, though they have smaller perihelia than the IOCs, spend the majority of their time between 
where Neptune and the known giant planets dominate and where the galactic tide becomes significant (50 to $1500 \mathrm{AU}$ ). Thus if there is any massive object still in this gravitationally depleted zone, it should show up as a signature on the orbits of these EDTNOs (Trujillo and Sheppard 2014). Their colors seem to be moderately red and thus similar to scattered disk objects (Sheppard 2010). New discoveries 2014 SR349 and 2013 FT28 are EDTNOs and have secure orbits as they have been observed for 2 and 4 oppositions, respectively. It is interesting to note that all of the known EDTNOs have perihelia near $45 \mathrm{AU}$ while both known IOCs have perihelia near 80 AU. It is low number statistics, so not much can be said, but their is obvious gaps between the extreme scattered objects, EDTNOs and IOCs in terms of perihelia (Figure 2).

The inclinations of the known EDTNOs and IOCs appear to be moderate with all but Sedna being between 17 and 26 degrees. We find the seven IOCs and EDTNOs have an average inclination of about 20 degrees. This is very similar to the inclination average of about 19 degrees found for the scattered disk objects by Gulbis et al. (2010).

\subsection{Argument of Perihelion Clustering}

Trujillo and Sheppard (2014) found that all ETNOs have similar arguments of perihelia and predicted a massive planet between some 200 to $1500 \mathrm{AU}$ was shepherding the ETNOs into these similar orbital angles. Interestingly, both of the new EDTNO discoveries 2014 SR349 and 2013 FT28 have arguments of perihelia in the same range as the other three known extreme detached objects (2010 GB174, 2004 VN112 and 2000 CR105) and two inner Oort cloud objects (2012 VP113 and Sedna) as well as almost all the ETNOs with perihelia greater than Neptune's (Figure 5). This strengthens the case that the clustering noticed in TS2014 is real and is statistically significant around the $6 \sigma$ level.

With the discovery of 2013 FT28, we now show there is a significant correlation between argument of perihelion and longitude of perihelion angles for ETNOs (Figure 4). ETNOs with longitude of perihelia between 0 and $120 \mathrm{deg}$ have arguments of perihelia between 280 and $360 \mathrm{deg}$. ETNOs with longitude of perihelia on the other side of the sky between 180 and $330 \mathrm{deg}$ have arguments of perihelia between 0 and $40 \mathrm{deg}$. Using a linear Pearson correlation, we find a Pearson correlation coefficient of 0.82 using a sample size of 15 ETNOs with $a>150 \mathrm{AU}$ and $q>35 \mathrm{AU}$, which gives a significance of the correlation between $\bar{\omega}$ and $\omega$ of around the $99.99 \%$ level. These two anti-clustered groupings of ETNOs can be further seen in their clustering in the Longitude of the Ascending Node angle (Figure 7) and orbit polar orientation angles, where the $\omega 0$ to $40 \mathrm{deg}$ group all come to perihelion above the ecliptic and the $\omega 280$ to $360 \mathrm{deg}$ group all below the ecliptic. 
These correlations are further evidence of an unknown massive planet, with the distant eccentric inclined orbit shown by BB2016 possible. This is because extreme eccentric objects with perihelia on opposite sides of the sky (180 degree longitude of perihelion differences) would approach the inclined planet at opposite points in their orbits (see Figure 12). Thus for the extreme objects to stay as far away as possible from the planet, they would prefer opposite ecliptic latitudes of the planet in order to avoid the planet above or below it (i.e. opposite argument of perihelion or orbit pole angles). Since the definition of the pole angle is based on the perihelion and the two groups have opposite perihelia locations, they would also have opposite pole angles or arguments of perihelia. The two ETNO groups seem to

show some sort of resonance behaviour with multiple orbital angles set to keep them away from the planet.

It is interesting to see that for the lower perihelion objects, $q<35 \mathrm{AU}$, where strong Neptune interactions should be at work, there is also clustering in the argument of perihelion for high semi-major axes ( $a>200 \mathrm{AU})$, but opposite or 180 degrees away from the ETNOs (Figure 6). This possible anti-correlation for $q<35$ AU objects from the $q>35$ AU objects with $a>150$ AU is currently unexplained.

\subsubsection{Argument of Perihelion Observational Biases}

There are two biases that could crop up in the argument of perihelion angle when discovering extreme objects. As noted in TS2014, surveys that are preferentially done near the ecliptic will be heavily biased towards finding objects with arguments of perihelion near 0 and 180 degrees as that is when those objects would be at perihelion and near the ecliptic and brightest. This 0 and 180 degree bias goes away as a survey covers more area away from the ecliptic and becomes minimal once a survey averages at least 10 degrees from the ecliptic. As our survey averages some 13 degrees off the ecliptic, there should be little to no 0 and 180 degree argument of perihelion bias. Further, even with the above 0 and 180 degree bias, one would still be equally likely to find ETNOs with arguments of perihelion near 180 degrees, where none or known, as near 0 degrees, where all are known. TS2014 show the above bias is not a factor in the observed clustering because objects found at high ecliptic latitudes, like most EDTNOs, will not have an observational bias in their argument of perihelion value. Both of the two new extreme detached objects reported here further refute any bias as both were found over five degrees from the ecliptic (17 and 7 degrees). We model observational biases specific to our survey below and show that the argument of perihelion clustering is extremely unlikely to be caused by chance.

A secondary argument of perihelion discovery bias exists if the survey fields are pref- 
erentially off the ecliptic in either the North or the South. Our survey reported here has mostly been obtained in the Southern Hemisphere as most observations have been from the CTIO 4m telescope with DECam that is located at about 30 degrees South latitude (Figure 3). As our fields are mostly South of the ecliptic we show this bias in Figure 11. That is, having mostly fields South of the ecliptic we are more sensitive to ETNOs with arguments of perihelion between 180 and 360 degrees, as they are more likely to come to perihelion and thus be brightest South of the ecliptic. But this secondary bias is not a major concern in showing that the ETNOs cluster in their arguments of perihelion with a range between about 290 and 40 degrees. That is because our survey, even with this secondary argument of perihelion bias, would be equally likely to find ETNOs between 90 and 270 degrees, where none are known, as 270 to 90 degrees, where all are known. Our ongoing survey is now also using the Subaru telescope in the North which should help remove this secondary bias from our observations. In addition, many of the ETNOs were discovered in surveys that did cover large Northern sky areas. We conclude there should be no major biases in the arguments of perihelion for the known ETNOs and thus they show strong clustering in their arguments of perihelion between about 290 and 40 degrees.

\subsection{Longitude of Perihelion Clustering}

Trujillo and Sheppard (2014) commented that both Sedna and 2012 VP113 are currently at similar locations on the sky and thus the IOCs may not be longitudinally symmetric. TS2014 further mentioned that if this was found to be true through the discovery of other IOC objects it would strongly constrain any formation scenario. This similar location on the sky seems unlikely to happen by chance and suggests a clustering in perihelion location. This was looked at in detail by Batygin and Brown (2016) to try and constrain what any distant planet's orbit may look like. But the similar location on the sky could be a selection effect as there is a possible bias in longitude of perihelion observations (Figure 14). This is because objects are most likely to be discovered at perihelion when they are brightest. The bias comes in because most surveys stay away from the galactic plane of the Milky Way to avoid confusion with background stars (around 5 to 8 hours and 16 to 19 hours in Right Ascension near the ecliptic, see Figure 3). Thus there are two main times of the year that

surveys for outer solar system objects are most efficient, which could possibly cause a bias in the longitude of perihelion discoveries.

Although the survey that found Sedna was all sky (Brown et al. 2004), the initial survey that found 2012 VP113 was not, and was only conducted in a similar location as Sedna (Trujillo and Sheppard 2014). This was a coincidence simply because that was the 
area observable for the first telescope observations of this survey when 2012 VP113 was found and thus it was highly biased in the longitude of perihelion angle of any discovered extreme objects. Thus the initial discovery of 2012 VP113 could not be used for the longitude of perihelion clustering as it was found in a highly biased location of the TS2014 survey. This is why TS2014 stated it was interesting that Sedna and 2012 VP113 shared similar locations on the sky but further discoveries would need to be made to look at this point further. Now with these new results presented here, the longitude of perihelion bias can be removed from 2012 VP113 as this survey has now covered similar amounts of sky on the other side of the galactic plane from Sedna's location and discovered new EDTNOs. Not including 2012 VP113, one extreme detached object was found on each side of the galactic plane during this survey. 2014 SR349, which has the fourth highest perihelion of any ETNO, including Sedna and 2012 VP113, was found with a similar argument of perihelion and longitude of perihelion as Sedna and 2012 VP113. 2013 FT28 was found on the other side of the sky with a longitude of perihelion some 180 degrees away from Sedna and 2012 VP113 (Figure 12).

It is interesting that 2013 FT28's longitude of perihelion is nearly 180 degrees away from the general center of the other longitude of perihelion extreme objects. BB2016 do suggest that if there is a giant planet on an eccentric orbit creating the longitude of perihelion clustering for extreme KBOs, there should also be objects 180 degrees away in longitude of perihelion. BB2016 predict such objects would be on much lower eccentricity orbits with high semi-major axes and thus much higher perihelia. In this regard, 2013 FT28 does not agree with the specific predictions made by BB2016. In a second paper, Brown and Batygin (2016) show a possible secondary longitude of perihelion clustering 180 degrees away from the main longitude of perihelion clustering for some simulations. This smaller population secondary longitude of perihelion clustering should generally take place at lower semi-major axes than most of the main clustering and it appears 2013 FT28 is in this secondary longitude of perihelion clustering.

\subsubsection{Stability of 2013 FT28}

There must be some objects that happen to be passing through the ETNOs region temporarily and are not on stable orbits, especially if there is an unknown massive planet beyond a few hundred AU scattering objects. The number of these passerbys is unknown. We looked deeper into 2013 FT28's orbit with some numerical simulations using the Mercury integrator (Chambers 1999). We cloned the orbit of 2013 FT28 a hundred times to cover the one sigma parameter space of its orbital uncertainty, which is low with about 3 years (2013-2016) of observational arc. Our simulations used the four giant planets and added the 
mass of the terrestrial planets to the Sun. The time step was 20 days and all integrations ran for 4 billion years.

We noticed that 2013 FT28's orbit is mostly stable but shows some movement in its semi-major axis over time, suggesting some interactions with the known giant planets, even though it has a high perihelion and large semi-major axis. In our numerical simulations about 30 percent of the time we found the semi-major axis of 2013 FT28 moved over 50 AU over the age of the solar system. Though none of the clones were lost from the solar system, significant semi-major axis movement suggests significant interactions with the known giant planets. In addition, the semi-major axis of the 2013 FT2 8 clones that didn't have moderate semi-major axis movement still showed some jumpiness in their semi-major axes suggesting some sort of resonant interactions with the giant planets (Figure 13).

This semi-major axis movement is unlike the 2014 SR349 numerical simulations that found all possible orbits to be stable in semi-major axis within a few AU over the age of the solar system (Figure 13). It is thus possible that 2013 FT28 has some sort of resonance interaction with the currently known giant planets, making its orbit less stable. The semimajor axis of the orbit of 2013 FT28 is known to within a few AU, but future observations might give an orbit that is more in the stable regime. We note that 2013 FT28 has a perihelion that is well beyond the 40-41 AU limit usually imposed for strong Neptune interactions for objects with high semi-major axes (Gomes et al. 2008; Brasser and Schwamb 2015).

\subsection{Stable and Unstable ETNOs}

BB2016 used only six of the twelve extreme objects identified in TS2014 to look for further evidence and possible orbits of this putative distant massive planet. BB2016 state in their section 2 that only stable objects over 4 billion years are useful in their analysis and is the reason they use only six of the 12 extreme objects. Stable to them was any extreme object that did not move significantly in its semi-major axis for 4 billion years. This limited BB2016 to the six extreme objects, which according to their Figure 1, were 2012 VP113, Sedna, 2010 GB174, 2004 VN112, 2000 CR105 and 2010 VZ98.

It seems odd for BB2016 to have included 2010 VZ98 as this object has one of the smallest semi-major axes and perihelia of the extreme objects (Table 3). We numerically integrated 2010 VZ98 and find it doesn't pass their basic criteria as it shows significant semimajor axis movement of over 100 AU within a few 100 Myrs (Figure 13). Thus as discussed above, the BB2016 statistical analysis should not have used 2012 VP113 as at that time the discovery of 2012 VP113 was from a longitude of perihelion biased survey. It also appears 
they should not have used 2010 VZ98 as the object has an unstable orbit (Table 3). This would leave only four extreme objects with stable orbits to test for longitude of perihelion clustering, which would have given a non statistically significant clustering result. In this paper we have removed the longitudinal biases from the discovery of 2012 VP113 and have found another stable EDTNO in 2014 SR349, which brings the usable stable EDTNOs and IOC objects back to six useful objects and a $0.7 \%$ chance of being clustered as observed in longitude of perihelion according to BB2016 statistics.

As we are using low number statistics, it is very important to determine which ETNOs are useful and which are not when looking for clustering. To further understand the dynamics of the known ETNOs, we numerically integrated all their nominal orbits for 4 billion years. In Table 3 we show the results of these numerical simulations. As expected, the IOC objects 2012 VP113 and Sedna were found to be very stable. The most distant perihelion EDTNOs were also very stable, 2010 GB174, 2014 SR349 and 2004 VN112. 2000 CR105 was stable, but showed spiked motion in its semi-major axis indicative of possible resonant interactions with the giant planets (Figure 13). This makes it questionable to use 2000 CR105 as a tracer for an unknown distant planet as significant interactions with the interior giant planets might cloud the dynamics of 2000 CR105. We find the extreme scattered object 2005 RH52 is also stable but again shows possible significant resonant interactions with the giant planets from spiked semi-major axis motion. We notice in Figure 13 that the larger the semi-major axis of the stable extreme objects, the larger the range in semi-major axis the object has over the age of the solar system ( \pm 5 AU for 2005 RH52 near 150 AU, \pm 8 AU for 2012 VP113 near $260 \mathrm{AU}, \pm 15$ AU for 2014 SR349 near 300 AU, \pm 20 AU for 2010 GB174 near 370 AU, and \pm 25 AU for Sedna near $500 \mathrm{AU})$.

We found all extreme objects with perihelia less than 38.5 AU showed semi-major axis variations greater than $100 \mathrm{AU}$ over a 1 billion year time-span, suggesting none may be good dynamical tracers of an unseen distant planet. Brown and Batygin (2016) further wrote about the extreme objects in a second paper. Here it is not obvious if they are using 2010 VZ98, but their Figure 1 shows they are using 2007 TG422 and 2013 RF98, both of which are also found to be unstable in our numerical integrations. 2013 RF98 has only a few month arc of observations and thus has a very large orbital uncertainty, but worse for 2013 RF98 is that its longitude of perihelion, though possibly similar to the other stable ETNOs, is best not to use. This is because 2013 RF98 was found by the Dark Energy Survey Collaboration et al. (2016) which has a strong longitude of perihelion angle bias since the survey only observes on the side of the sky that Sedna and 2012 VP113 are in, where objects with these longitude of perihelion angles would be brightest and easiest to find. Brown and Batygin (2016) appear most interested in ETNOs with $a>250 \mathrm{AU}$ and is the reason they highlight 2007 TG422 and 2013 RF98. We find 2013 RF98 and 2007 TG422 are unstable from Neptune 
perturbations over 10 Myr times scales, but because of their very distant semi-major axes the hypothesized more distant planet could have a much stronger effect on these orbits and thus dominate over any Neptune or Uranus perturbations. If this is the case, than 2013 FT28 should also be used as it is much more stable then these other objects. If the clustered unstable objects are lost on short time-scales, their must be a reservoir somewhere replacing them.

\subsubsection{Statistics of ETNO Clustering}

It is important to determine which ETNOs are best to use in low number statistical analyses. 18 of 19 extreme objects shown in Table 3, stable or unstable, show the same clustering in argument of perihelion between 290 and 40 degrees as first identified in TS2014. Only 2013 FS28 does not show this clustering, but 2013 FS28 also has the second lowest perihelion ( $q \sim 34 \mathrm{AU}$ ) of any ETNO and thus likely has strong interactions with Neptune and thus we suggest only using objects with $q>35$, though $q>40$ AU may be more appropriate to further remove Neptune interactions. We further have shown that there is little to no bias in the clustering of the argument of perihelion from discoveries and have shown that strangely, the objects with $a>200$ AU and $q<35$ AU show a 180 degree opposite clustering for the argument of perihelion angles (100 to 200 degrees). Finally, we also showed that there is a correlation between the argument of perihelion and longitude of perihelion for ETNOs. The unstable objects seem to demonstrate the same clustering properties as the stable objects. This suggests that the unstable objects also provide clues to the dynamical processes in the $50 \mathrm{AU}$ to $1500 \mathrm{AU}$ outer solar system regime and that the unseen planet's interactions might dominate over Neptune interactions.

For the longitude of perihelion clustering 12 of the 19 extreme objects in Table 3 show clustering in the main longitude of perihelion clustering between 0 and 130 degrees. This is less distinct than the clustering in argument of perihelion and thus one might want to limit the ETNOs used to see if the clustering is stronger or weaker. BB2016 talk about using only stable objects or objects with higher semi-major axes of 250 or $350 \mathrm{AU}$ instead of $150 \mathrm{AU}$. Using only these objects, the statistics get better, but the number of objects is low (6 of 9 for mostly stable ETNOs, 5 of 6 for completely stable ETNOs, 7 of 8 for $a>250$ ETNOs, 5 of 5 for completely stable, $a>250$ AU and $q>45$ AU ETNOs). In the most stringent case there are only 5 useful objects and thus the statistics are almost but not quite significant at the 3 sigma level. Finding more of these ETNOs with very high perihelia $q>45 \mathrm{AU}$ and semi-major axes $a>250 \mathrm{AU}$ is required to fully confirm that the longitude of perihelion clustering is real. The longitude of perihelion clustering also gets a 
little uncertain since Brown and Batygin (2016) showed objects could be in a secondary antior 180 degree opposite clustering in longitude of perihelion, which we believe 2013 FT28 is part of. With this, the range of acceptable longitude of perihelia for ETNOs becomes much wider and thus harder to look for significant outliers, but including both the primary and 180 degree secondary longitude of perihelion angles, we find for $a>250$ AU that 8 of 8 objects are clustered in longitude of perihelion.

Clustering in the Longitude of Ascending node for high perihelion objects is also apparent for the two different longitude of perihelion clusters (Figure 7). Our numerical simulations show that the argument of perihelion cycles through all angles about twice as fast as the longitude of perihelion angle for each extreme object when only including the known major planets. As noted earlier, the argument of perihelion appears to be significantly correlated with the longitude of perihelion for all ETNOs.

\subsection{Likelihood of Orbital Clustering Through An Observational Bias Simulator}

In section 3 above we described the basics of our observational simulator. In our real observational survey data, we found no IOCs nor EDTNOs with $40 \mathrm{deg}<\omega<290 \mathrm{deg}$ nor any with $230 \mathrm{deg}<\Omega<360 \mathrm{deg}$ and 1 IOC and 2 EDTNOs outside of these ranges. We find the odds of detecting 1 IOC and 2 EDTNOs in the $290 \mathrm{deg}<\omega<40 \mathrm{deg}$ regime is $10 \%$ from our survey in the radially symmetric case. The odds of detecting 1 IOC and 2 EDTNOs with $0 \mathrm{deg}<\Omega<230 \mathrm{deg}$ in the radially symmetric case is $18 \%$. Assuming $\Omega$ and $\omega$ are statistically uncorrelated results in a $1.8 \%$ probability that this could happen by chance from our survey detections of the IOC and 2 EDTNOs alone if the populations are radially symmetric.

Both Sedna and 2012 VP113 were found in similar sky locations as reported in TS2014. Sedna was discovered at a right ascension of 03:13 hours and a declination of $+05: 47$ degrees and 2012 VP113 discovered at a right ascension of 03:23 hours and a declination of $+01: 12$ degrees, less than 6 degrees apart. We quantified how many IOCs would be detected in our simulation within 6 degrees of Sedna's discovery location given our observed fields and survey methodology. The probability of this occurring by chance in a symmetric population given our survey field distribution is less than 1 in 1000. Thus, this provides further evidence that the IOCs are in an longitudinally asymmetric population as first suggested in TS2014.

We test for observational evidence for longitude of perihelion by simulating a population that is uniform in terms of the angular elements $\omega$ and $\Omega$ (and thus longitude of perihelion 
$\bar{\omega}=\omega+\Omega)$. Then we estimate the probability that the true distribution of objects violates the discovery statistics expected under these assumptions. Given the uniform $\omega$ and $\Omega$ case, one would expect 35 of 130 detected simulated objects to fall in the $0<\bar{\omega}<130$ deg range given our true detection statistics. In our survey, 2 extreme objects fell into this range, 2012 VP113 and 2014 SR349 and one outside 2013 FT28. If we reject 2013 FT28 from this sample because it may have significant Neptune interactions or because it may lie in the secondary anti-longitude of perihelion clustering, the probability of this longitude of perihelion range happening by chance in our survey alone is $7 \%$, which is not statistically significant.

\subsubsection{Biases Of Other Discovered Extreme TNOs}

Other surveys which have detected extreme TNOs may have similar biases to our survey if they have covered a large amount of sky generally distributed throughout the year in terms of search date. Under this assumption, there are 5 known stable IOCs and EDTNOs with $a>250$ AU that fall into the $0<\bar{\omega}<130$ deg range (2012 VP113, Sedna, 2010 GB174, 2014 SR349 and 2004 VN112: Table 3). The probability of all 5 known objects having a clustered $\bar{\omega}$ between 0 and 130 degrees by chance is then $(35 / 130)^{5}=1.4 \times 10^{-3}$ which is around $3 \sigma$ assuming Gaussian statistics.

But other surveys that have found extreme TNOs do not have the minimal biases as our survey, which makes the assumption of similar biases untrue. As detailed earlier, 2012 VP113 and 2014 SR349, found in this work, have minimal to no bias in longitude of perihelion. Sedna, found in a mostly all sky survey, also likely has no significant longitude of perihelion bias (Brown et al. 2004). But 2004 VN112 was found in the ESSENCE supernova survey that was mostly obtained between October and December, which is similar to the location on the sky of Sedna and 2012 VP113 (Becker et al. 2008). So 2004 VN112 has a strong longitude of perihelion bias, though should have no significant argument of perihelion bias as it was a survey well off the ecliptic. 2010 GB174 was found in a small limited survey well off the ecliptic that was focused near the Virgo cluster around 12 to 13 hours in RA (Chen et al. 2013). This is on the opposite side of the Milky Way than Sedna and 2012 VP113, but the limited survey area makes it questionable how to handle the bias in longitude of perihelion for 2010 GB174.

Some of the other objects shown in Table 3 that we find are more unstable do have minimal longitude of perihelion discovery biases. 2000 CR105, which was found in the Deep Ecliptic Survey (DES) that observed at all times of the year (Elliot et al. 2005), 2005 RH52 from the CFHT Ecliptic Plane Survey (Petit et al. 2011) and 2013 GP136 found in the Outer Solar System Origins Survey (Bannister et al. 2016). As mentioned earlier, 2013 RF98 has 
a strong longitude of perihelion bias as the Dark Energy Survey that found it only observes

the sky near Sedna and 2012 VP113 (Dark Energy Collaboration et al. 2016). Most of the other objects shown in Table 1 do not have known discovery biases.

Thus with the new data reported here, it appears that the longitude of perihelion clustering for the ETNOs is real and there are two distinct anti-clusters, as long as assumptions about other survey biases not being important are true, stable and unstable objects are equally reliable tracers, and one places 2013 FT28 into the secondary anti-longitude of perihelion clustering region. Further unbiased extreme TNO discoveries need to be made to confirm the longitude of perihelion clustering.

\subsection{Extreme TNOs Orbital Period Ratios}

Malhotra et al. (2016) found that four of the EDTNOs (Sedna, 2010 GB174, 2004 VN112 and 2012 VP113) would have N/1 or N/2 period ratios with a hypothetical massive planet with a semi-major axis around 665 AU. These period ratios could be an indication of the EDTNOs being in some sort of mean motion resonance with a distant massive planet. The two new ETNOs reported here, 2014 SR349 and 2013 FT28, have fairly similar semimajor axes, eccentricities and inclinations, though they have opposite longitude of perihelia as discussed above. 2014 SR349 and 2013 FT28 would have about 4880 and 5460 year orbital periods and are not similar to any of the other known EDTNOs. The new EDTNOs would have an orbit period ratio of about $3.5 \pm 0.3$ and $3.13 \pm 0.1$ respectively, if the hypothetical unknown distant massive planet had a period of 17116 years. Thus 2014 SR349 could be near the $7 / 2$ resonance, though the uncertainty in the orbit is still too large to make any definitive statements. 2013 FT28 has a better constrained orbit and isn't near any of the major resonances, though the $3 / 1$ isn't too far off.

\section{Population Statistics}

\subsection{Extreme Detached TNOs Population Statistics}

We considered two possible EDTNO populations: one with a uniform distribution of the angular orbital elements argument of perihelion and longitude of ascending node, and a second with a constrained set of angular orbital elements noted by TS2014 and BB2016. For the constrained angular case, we used an argument of perihelion range of $-80 \mathrm{deg}<$ $\omega<45 \mathrm{deg}$ and a longitude of perihelion range of $0 \mathrm{deg}<\bar{\omega}<130 \mathrm{deg}$. The longitude of ascending node was then computed from $\Omega=\bar{\omega}-\omega$. 
To estimate the population of EDTNOs we used a radially symmetric and asymmetric population described in Table 5 based on our 2 detections of EDTNOs (2014 SR349 and 2013 FT28) and the results of our observational bias simulator (Figure 14). For the asymmetric case, we find that the total number of EDTNOs in the radius range $100 \mathrm{~km}<r<4000 \mathrm{~km}$ to be 1800 and the total mass to be $3.8 \times 10^{22} \mathrm{~kg}$ ( $\sim 1 / 150$ of an Earth mass) assuming a differential power law of $q^{\prime}=-5$ (Figure 15). Due to the number of assumptions in our simulation and the small number of detections, these quantities are only order of magnitude estimates. As more EDTNOs are discovered in our ongoing survey, we will be able to pin down the total mass of the EDTNOs with much more accuracy. Population estimates for other assumptions, such as $q^{\prime}=-4$ and symmetric populations, can be found in Table 5 .

\subsection{Updated Inner Oort Cloud Object Population Statistics}

In TS2014 we gave IOC population statistics assuming a symmetric population, but stated in TS2014 that 2012 VP113 was found at a similar sky position as Sedna and thus the population might not be longitudinally symmetric on the sky (i.e. there could be longitude of perihelion clustering). The IOC population would be smaller than that reported in TS2014 if the IOC objects are not uniformly distributed across the sky. This survey has now covered almost 20 times more sky area than analyzed in TS2014 and thus the population statistics of the IOC objects is likely significantly less than reported in that paper as no new IOC object has been found in a much more uniform sky survey. Our survey as reported in TS2014 was longitudinally biased and thus it was not useful to determine if the IOCs where longitudinally asymmetric. Our much larger survey reported here is not longitudinally biased and thus we can report revised population statistics for the IOCs assuming an asymmetric population in longitude.

Our current survey found one inner Oort cloud object ( $q>50 \mathrm{AU}$ and $a>150 \mathrm{AU}$ ), 2012 VP113. For our model populations we restricted our minimum perihelion to 75 AU since the two known IOCs, Sedna and 2012 VP113, both have perihelia above this range, which might suggest an inner edge around 75 AU (Trujillo and Sheppard 2014). We assumed that the IOCs also have constrained angular orbital elements, similar to the EDTNOs (Table 5). From the detection of 2012 VP113 and the survey fields we have covered, we find the number of bodies in the radius range $100 \mathrm{~km}<r<4000 \mathrm{~km}$ to be $2 \times 10^{4}$ and a total mass of $4 \times 10^{23}$ $\mathrm{kg}$ or $\sim 1 / 10$ of an Earth mass (Figure 15). We expect there should be a few objects larger than Pluto in the IOC. These numbers are very dependent on the eccentricity distribution of the IOCs. For instance, we are very insensitive to the most eccentric IOCs since they spend most of their time at aphelion and too faint to be detected. Thus, the eccentricity 
distribution and semi-major axis distribution have a large impact on the total mass and number of bodies estimated. We have chosen fairly conservative numbers for these, so if anything, the estimated population of IOCs is likely to increase with further observational constraints rather than decrease.

\subsection{The Inner Oort Cloud Inner Edge}

One important question is whether the EDTNOs and the IOCs could be drawn from the same population. Currently, the two known IOCs Sedna and 2012 VP113 have perihelia greater than 75 AU. In contrast, the EDTNOs all have perihelia less than 50 AU. The main observational question is then whether a single set of population-wide parameters could encompass both populations, or whether there is a gap or dearth of objects in the 50 AU to 75 AU regime. This was simulated by TS2014 who determined that the paucity of objects in the $50 \mathrm{AU}$ to $75 \mathrm{AU}$ regime was most likely not observational bias using both the original survey that found Sedna and their survey. We repeat this simulation here for only the survey described in this paper. This is a more thoroughly characterized survey and represents and additional factor of $\sim 20$ more sky coverage than our survey in TS2014.

If the EDTNOs and IOCs were drawn from the same population, one would expect large semi-major axis objects to be found with perihelion in the $50 \mathrm{AU}$ to $75 \mathrm{AU}$ regime. In our survey simulator, we find that $71 \%$ of detections should occur in the $50 \mathrm{AU}$ to $75 \mathrm{AU}$ range. Since we found 2 EDTNOs and 1 IOC object, none of which had perihelia in the 50 to 75 AU range, the probability of this occurring by chance is about $2 \%$. It is not clear whether including the extreme scattered objects 2013 UH15, 2013 FS28 and 2014 SS349 would be appropriate, but if they were included the probability would drop to $0.1 \%$ and thus be statistically significant above the $\sim 3 \sigma$ level using our survey data alone.

Other surveys have found the other 19 known ETNOs, none of which have perihelion in the $50 \mathrm{AU}$ to $75 \mathrm{AU}$ range. If other surveys have similar biases to our own, the probability of this occurring by chance is roughly $0.29^{19}=6 \times 10^{-11}$ or about $7 \sigma$ assuming Gaussian statistics.

\subsection{Cumulative Luminosity Function}

The Cumulative Luminosity Function (CLF) describes the sky-plane number density of objects brighter than a given magnitude. The CLF can be described by

$$
\log \left[\Sigma\left(m_{r}\right)\right]=\alpha\left(m_{r}-m_{o}\right)
$$


where $\Sigma\left(m_{r}\right)$ is the number of objects brighter than $m_{r}, m_{o}$ is the magnitude zero point, and $\alpha$ describes the slope of the luminosity function. There is no known CLF for the IOC objects or EDTNOs as few are known. We here attempt a crude estimate based on our detection of one IOC object (2012 VP113) and two EDTNOs (2014 SR349 and 2013 FT28). The CLF for these objects is shown in Figure 16. The slope for the CLF for the IOC objects appears consistent with that found for the moderate sized Kuiper Belt objects $(\alpha \sim 0.6$ : Fraser $\&$ Kavelaars 2008; Fuentes \& Holman 2008), but just shifted downwards $\left(m_{o} \sim 28\right)$ to adjust for the lower number of objects per brightness bin on the sky.

\section{Additional Interesting Objects Discovered}

\subsection{Outer Oort Cloud Discovery}

The first object that enters into the outer Oort cloud yet has perihelion greater than Neptune was discovered as part of this survey. 2014 FE72 has a semi-major axis around 2155 AU and an aphelion distance of some 4000 AU. Thus outside forces such as the galactic tide and stellar perturbations have likely influenced the orbit of 2014 FE72 over time (Duncan et al. 2008; Soares and Gomes 2013; Kaib and Quinn 2009). The moderate perihelion of 2014 FE72 around 36 AU suggests that its highly elliptical orbit is from past Neptune encounters, but its longitude of perihelion $\bar{\omega}=111$ degrees is similar to the other known high semi-major axes objects $(0<\bar{\omega}<130$ degrees $)$. This could suggest that the possible massive unknown outer planet is shepherding 2014 FE72 similarly as the other EDTNOs and IOCs. Since 2014 FE72's inclination is only around 20 degrees, it is similar to the other extreme objects.

\subsection{The Curious Case of 2014 SS349}

The object 2014 SS349 is a borderline extreme object as it has a semi-major axis just below the ETNOs cutoff of 150 AU. The strength of a Neptune resonance decreases as the semi-major axis of an object increases and thus the large semi-major axes of the ETNOs and IOC objects suggest they are not strongly perturbed by resonances from Neptune and this may be the case of 2014 SS349 as well. 2014 SS349 has a fairly large inclination of 48 degrees, suggesting its orbit is modified by the Kozai resonance. Though its perihelion is now about 45.5 AU, it could travel much closer to Neptune's orbit through the Kozai mechanism alone or in combination with some Neptune mean motion resonance interaction in the past (see Gomes et al. 2008 or Kaib \& Sheppard 2016). Thus 2014 SS349 is probably more like the high perihelion objects with moderate eccentricities and semi-major axes discussed in 
Sheppard et al. (2016) that likely have a different origin and history than the ETNOs.

\subsection{New Extreme Scattered Disk objects}

The objects 2013 UH15 and 2013 FS28 have large semi-major axes, but their perihelia are too close to Neptune $(q<35$ AU) to be considered EDTNOs like 2014 SR349 and 2013 FT28. Both objects were lost within the age of the solar system during numerical integrations through Neptune interactions, so the most likely explanation for these extreme scattered objects is that they are related to scattered disk objects like 1996 TL66 (Luu et al. 1997; Duncan and Levison 1997; Gomes et al. 2008). The new discovery 2013 FS28 is the only ETNO of 19 known that does not follow the argument of perihelion clustering for objects with $a>150 \mathrm{AU}$ and perihelia greater than Neptune. 2013 FS28 has the second lowest perihelion of any ETNO so likely has strong Neptune interactions.

\subsection{Possible High Order Neptune Mean Motion Resonances}

2014 SW349, 2014 FJ72 and 2014 FL72 appear to have similar orbits (Table 4). Their moderately high perihelia of 38 to 39 AU makes them significantly far from Neptune to have strong Neptune interactions. They all could be in some high order mean motion resonance with Neptune, or could just be typical scattered disk objects. Though they have relatively high perihelia, they are not extreme because of their moderate semi-major axes. All three objects appear stable for the age of the solar system in our numerical simulations.

Objects 2014 SZ349 and 2014 SD350 are near the 13:3 Neptune mean motion resonance. Both have perihelia fairly close to Neptune, which allows Neptune to strongly influence their orbits. Neither 2014 SD350 or 2014 SZ349 were stable for the age of the solar system in our numerical simulations.

\subsection{Other Discoveries}

This survey has discovered some of the most distant objects ever observed in the solar

system. The TNO 2013 FY27 was found to be one of the most distant and one of the brightest outer solar system objects discovered in this survey. The 22 magnitude r-band magnitude and 80.5 AU distance of 2013 FY27 give the object an absolute magnitude of about 2.9. This makes 2013 FY27 one of the top ten intrinsically brightest TNOs and thus it could be a top ten largest TNO as well. Though the diameter and albedo are unknown, 
assuming an albedo of 0.1 yields a diameter around $1000 \mathrm{~km}$.

A new possible bright Haumea family member was discovered as well, 2014 FT71 ( $a=$ 43.8 AU, $e=0.14$, and $i=27.9 \mathrm{deg}$ ), based on orbital similarity to other known members of the Haumea family (Ragozzine and Brown 2007). If true, infrared spectroscopy of this object should show very strong water ice absorption (Brown et al. 2007; Snodgrass et al. 2010; Trujillo et al. 2011).

One well know main belt asteroid, (62412) 2000 SY178, was found to show a tail during our survey observations (Sheppard and Trujillo 2015). This object is now classified as an active asteroid (Jewitt 2012; Hsieh et al. 2015) and was found to be part of the Hygiea outer main belt asteroid family with a fast rotation period. Further details about active asteroid 62412 are available in Sheppard and Trujillo (2015). Through the survey, comet C/2014 F3 (Sheppard-Trujillo) was discovered to be one of the most distant active comets ever observed at 13 AU. Its perihelion is near Jupiter, suggesting strong interactions with Jupiter. Finally, 2007 TY430 was discovered and found to be an ultra-red, equal-sized, wide binary Kuiper Belt object in the Neptune 3:2 mean motion resonance. This makes 2007 TY430 the only known ultra-red, equal-sized, wide binary known outside of the main classical Kuiper Belt (see Sheppard et al. 2012).

For completeness, we here list all the objects discovered beyond 50 AU during our survey to date. The most updated orbits for the objects can be found at the Minor Planet Center. For 2012 VP113 see Trujillo and Sheppard (2014). For 2014 FZ71, 2013 FQ28, 2015 GP50, 2014 FC69 and 2012 FH84 see Sheppard et al. (2016). Other discoveries beyond 50 AU with decent orbital elements are: 2013 FY27, 2013 FT28, 2013 FS28, 2013 UH15, 2014 FE72, 2014 SR349, 2014 SS349, 2014 FJ72, 2014 FL70, 2011 GM89, 2012 FN84, 2012 OL6, 2013 UJ15, 2013 UK15, 2014 FF72, 2014 FL72, 2014 FM72, 2014 FH72, 2014 FG72, 2014 FK72, 2014 SJ350, 2014 SV349, 2014 SG350, 2014 SK350, 2014 SD350, 2014 SW349, 2014 SY349, 2014 SX349, 2014 SB350, 2014 SL350, 2014 SE350, 2014 SC350, 2014 SM350, 2014 SF350, 2014 ST349, 2014 SU349, 2014 SZ349, 2015 GR50, 2015 GS50, and 2015 GQ50. A few objects were not followed after about 1 month of observation arc as their orbits didn't look extreme or interesting or were found to be at less than 50 AU distance after recovery: 2012 FM84, 2013 VJ24, 2014 FN72, 2014 FP72, 2014 FO72, 2014 SA350, and 2014 SH350. Finally, there were two objects that were in the noise at discovery and appear to be beyond $50 \mathrm{AU}$, but were too faint to easily recover: D359c02 (found on field decam359 on chip 02) around 24.5 mags near 65 AU at 13:24:11 hours and -13:26:48 degrees on March 17.283, 2013 and D364c08 24.5 mags around 70 AU at 13:24:51 hours and -24:20:08 degrees on March 17.301, 2013. 


\section{Finding a Super-Earth to Neptune Mass Planet in the Outer Solar System}

We also simulated our survey's sensitivity to finding the distant massive planet predicted by TS2014 with the recently hypothesized rudimentary orbit described by BB2016. Our survey has attempted to obtain uniform sky coverage in both ecliptic latitude and longitude to prevent biases in the orbital parameters of discovered objects. To this end, we find that our survey would not detect such a distant eccentric planet some $99 \%$ of the time. This is mostly because the possible hypothesized planet's large eccentricity implies that it spends most of its time near aphelion, which would make it very faint and a significant fraction of its time in the northern sky if it is anti-aligned with the ETNOs. Most of the sky area covered in this work was in the southern sky due to our use of DECam at CTIO, which has a latitude near 30 degrees South. Our continuing survey is now covering large portions of the Northern sky using HyperSuprimeCam on the Subaru telescope and will be detailed in future work. Figure 17 shows that even a very massive 10 Earth mass planet beyond a few hundred AU would likely go undetected in most surveys to date as its r-band magnitude would be fainter than 24th magnitude with moderate to dark albedo.

In this work we have used IOC to refer to bodies with extremely high perihelia $(q>75$ $\mathrm{AU})$ for consistency with past works. We note that if the distant massive planet hypothesis is correct, it implies that the IOC population is not in fact primordial, nor is it directly related to the Oort cloud. What we have been calling the IOC would actually be a highly evolved dynamical population that is strongly influenced by the distant massive planet. Thus, a different nomenclature should be used when discussing bodies like Sedna and 2012 VP113. Calling IOC objects as just simply extreme detached objects seems most logical as it would appear both these populations would be directly related in the Planet X/Planet 9 regime. Since the unseen planet has not been discovered and no such nomenclature has been agreed upon, it is an open discussion. Calling the objects most perturbed by the putative distant planet Sednoids could also be logical after the brightest and first obvious member of this population.

\section{Summary}

We have covered about 1080 square degrees of sky to over 24th magnitude searching for objects beyond the Kuiper Belt edge. Unlike most faint outer solar system surveys, our survey has fairly uniform sky coverage in ecliptic longitude and southern ecliptic latitudes between about 5 and 20 degrees. Thus our survey should have little to no bias in the longitude of perihelion of discovered objects. The average ecliptic latitude was some 13 degrees from the ecliptic plane, which would limit the argument of perihelion bias during 
discovery that previous ecliptic plane surveys could have. Several new extreme objects with perihelia greater than $40 \mathrm{AU}$ and semi-major axes greater than $150 \mathrm{AU}$ were discovered.

1) Newly discovered extreme detached trans-Neptunian object 2014 SR349 has a semimajor axis around $288 \mathrm{AU}$ and a distant perihelion of almost 48 AU. 2014 SR349 was found to have a stable orbit over the age of the solar system and a argument of perihelion and longitude of perihelion similar to the other known extreme trans-Neptunian objects and inner Oort cloud objects. Thus 2014 SR349 continues to show the orbital clustering of extreme trans-Neptunian objects first identified and explained as likely caused by a distant massive planet between about 200 to 1500 AU in the outer solar system in Trujillo and Sheppard (2014).

2) 2013 FT28 is also a newly found extreme detached trans-Neptunian object with a similar semi-major axis as 2014 SR349 and slightly lower perihelion of 43.6 AU. The argument of perihelion for 2013 FT28 is consistent with the clustering of argument of perihelia for extreme objects, but its longitude of perihelion is not as it is about 180 degrees away from the other extreme detached objects. 2013 FT28 appears to be the first high semi-major axis object within the secondary longitude of perihelion clustering that could be 180 degrees from the main clustering discussed in Brown and Batygin (2016) for an eccentric, inclined distant massive planet. 2013 FT28 is the highest perihelion object with a semi-major axis above 250 $\mathrm{AU}$ to show some orbit instability and thus demonstrates significant Neptune interactions are possible even for objects with perihelia around 43.6 AU.

3) The argument of perihelion clustering for extreme objects is strongly statistically significant at about the $6 \sigma$ level with all 15 objects with $q>35$ and $a>150$ AU having arguments of perihelion between 290 and 40 degrees. In addition to the extreme objects with perihelia greater than 35 AU having argument of perihelia clustered in the range between about 290 and 40 degrees, we find the opposite is true for objects with perihelia less than $35 \mathrm{AU}$ and $a>200 \mathrm{AU}$. These high semi-major axis trans-Neptunian objects with perihelia less than 35 AU have arguments of perihelia that cluster in the range 100 to 200 degrees. This reverse clustering is not yet explained.

4) The longitude of perihelion clustering is not as robust as the argument of perihelion clustering. Past statistical analyses on the possible longitude of perihelion clustering of extreme trans-Neptunian objects used objects with biased longitude of perihelion discoveries. With the new results from this work, we show the longitude of perihelion clustering is likely real. With the discovery of 2013 FT28, we find there are two longitude of perihelion clusters for extreme objects about 180 degrees apart.

5) The longitude of perihelion is significantly correlated with the argument of perihelion 
at the $99.99 \%$ level for ETNOs as those with $\bar{\omega}=0$ to 130 have $\omega=280$ to 360 and come to perihelion below the ecliptic while those with $\bar{\omega}=180$ to 340 have $\omega=0$ to 40 degs and come to perihelion above the ecliptic. We find these two groups also have correlated longitude of ascending node clustering and orbit pole angle clustering. Thus we find two distinct ETNO clustering groups that are anti-aligned with each other. This correlation is further evidence of an unknown massive planet in the outer solar system. This is because extreme eccentric objects with perihelia on opposite sides of the sky (180 degree longitude of perihelion differences) would approach the planet at opposite points in their orbits. Thus for the extreme objects to stay away from the planet, they would prefer opposite ecliptic latitudes of the planet in order to avoid the planet (i.e. opposite argument of perihelia or orbit pole angles).

6) 2014 SS349 is an object with a high perihelion $(q=45.5 \mathrm{AU})$, fairly high semi-major axis $(143 \mathrm{AU})$ and high inclination ( $i=48$ degrees). It is a borderline extreme detached object like 2014 SR349 and 2013 FT28, but its much higher inclination and lower semi-major axis suggests it is likely to be much more influenced by the Kozai resonance. The dynamics of this object need to be further explored, but it is not considered an extreme object.

7) 2014 FE72 has a semi-major axes around 2155 AU and a aphelion around 4000 AU and likely experiences significant perturbations from outside forces such as the galactic tide. This makes 2014 FE72 the first outer Oort cloud object discovered that has a perihelion beyond Neptune. This object likely interacted with Neptune in the past to obtain such a distant eccentric orbit. Surprisingly, the longitude of perihelion of 2014 FE72 is similar to the other ETNOs with very large semi-major axes.

8) The cumulative luminosity function for the inner Oort cloud objects and extreme objects suggests a similar slope as the Kuiper Belt object population. Based on the area and objects discovered in this survey, we estimate about 21000 inner Oort cloud objects $(q>75$ $\mathrm{AU})$ and 1800 extreme detached trans-Neptunian objects $(40<q<50 \mathrm{AU})$ exist larger than $100 \mathrm{~km}$ in size assuming an asymmetric population with most coming to perihelion between about 2 and 7 hours in Right Ascension.

9) The inclinations of the EDTNOs and IOCs are moderate with all but Sedna being between 17 and 26 degrees. Their average inclination is about 20 degrees, which is very similar to the scattered disk population.

10) We note that if there is a massive planet shepherding the inner Oort Cloud objects and extreme detached objects in their orbits, these two populations are likely to be from the same source. Further, the orbits of the inner Oort cloud objects would not be primordial or directly related to the Oort cloud and thus the name inner Oort cloud objects does not seem 
appropriate.

\section{Acknowledgments}

This project used data obtained with the Dark Energy Camera (DECam), which was constructed by the Dark Energy Survey (DES) collaborating institutions: Argonne National Lab, University of California Santa Cruz, University of Cambridge, Centro de Investigaciones Energeticas, Medioambientales y Tecnologicas-Madrid, University of Chicago, University College London, DES-Brazil consortium, University of Edinburgh, ETH-Zurich, University of Illinois at Urbana-Champaign, Institut de Ciencies de l'Espai, Institut de Fisica d'Altes Energies, Lawrence Berkeley National Lab, Ludwig-Maximilians Universitat, University of Michigan, National Optical Astronomy Observatory, University of Nottingham, Ohio State University, University of Pennsylvania, University of Portsmouth, SLAC National Lab, Stanford University, University of Sussex, and Texas A\&M University. Funding for DES, including DECam, has been provided by the U.S. Department of Energy, National Science Foundation, Ministry of Education and Science (Spain), Science and Technology Facilities Council (UK), Higher Education Funding Council (England), National Center for Supercomputing Applications, Kavli Institute for Cosmological Physics, Financiadora de Estudos e Projetos, Fundao Carlos Chagas Filho de Amparo a Pesquisa, Conselho Nacional de Desenvolvimento Cientfico e Tecnolgico and the Ministrio da Cincia e Tecnologia (Brazil), the German Research Foundation-sponsored cluster of excellence "Origin and Structure of the Universe" and the DES collaborating institutions. Observations were partly obtained at Cerro Tololo Inter-American Observatory, National Optical Astronomy Observatory, which are operated by the Association of Universities for Research in Astronomy, under contract with the National Science Foundation. C.T. is supported by the Gemini observatory, which is operated by the Association of Universities for Research in Astronomy, Inc., on behalf of the international Gemini partnership of Argentina, Australia, Brazil, Canada, Chile, the United

Kingdom, and the United States of America. This research was funded by NASA Planetary Astronomy grant NNX12AG26G and NN15AF44G. This paper includes data gathered with the 6.5 meter Magellan Telescopes located at Las Campanas Observatory, Chile.

\section{REFERENCES}

Bannister, M, Kavelaars, J., Petit, J. et al. 2016, arXiv:1511.02895.

Batygin, K. \& Brown, M. 2016, AJ, 151, 22.

Becker, A., Arraki, K., Kaib, N., et al. 2008, 682, L53. 
Beust, H., 2016 A\&A, 590, 2.

Bottke, W., Durda, D., Nesvorny, D., et al. 2005, Icarus, 175, 111

Brasser, R., Duncan, M., \& Levison, H. 2006, Icarus, 184, 59.

Brasser, R., Duncan, M., Levison, H., Schwamb, M. \& Brown, M. 2012, Icarus, 217, 1.

Brasser, R. \& Schwamb, M. 2015, MNRAS, 446, 3788.

Bromley, B. \& Kenyon, S. 2014, ApJ, 796, 141.

Brown, M., Trujillo, C. \& Rabinowitz, D. 2004, ApJ, 617, 645.

Brown, M., Barkume, K., Ragozzine, D. \& Schaller, E. 2007, Nature, 446, 294.

Brown, M. \& Batygin, K. 2016, ApJ, 824, 23.

Brown, R. \& Firth, J. 2016, MNRAS, 456, 1587.

Chambers, J. 1999, MNRAS, 304, 793.

Chen, Y., Kavelaars, J., Gwyn, S., Ferrarese, L., Cote, P., Jordan, A., Suc, V., Cuillandre, J., \& Ip, W. 2013, ApJ, 775, L8.

Chiang, E., Jordan, A., Millis, R. et al. 2003, AJ, 126, 430.

Cowan, N., Holder, G. \& Kaib, N. 2016, ApJ, 822, 2.

Dark Energy Survey Collaboration et al., 2016, MNRAS, 460, 1270.

de la Fuente Marcos, C. \& de la Fuente Marcos, R. 2014a, MNRAS, 443, 59.

de la Fuente Marcos, C. \& de la Fuente Marcos, R. 2014b, Ap\&SS, 352, 409.

de la Fuente Marcos, C. \& de la Fuente Marcos, R. 2016, MNRAS, 459, 66.

Dressler, A., Bigelow, B., Hare, T., et al. 2011, PASP, 123, 288.

Duncan, M., Brasser, R., Dones, L., and Levison, H. 2008, in The Solar System Beyond Neptune, eds. M. Barucci, H. Boehnhardt, D. Cruikshank and A. Morbidelli (Tucson: Univ of Arizona Press), 315-331.

Elliot, J., Kern, S., Clancy, K., et al. 2005, AJ, 129, 1117.

Fienga, A., Laskar, J., Manche, H. \& Gastineau, M. 2016, A\&A, 587, 8.

Flaugher, B., Diehl, H., Honscheid, K., et al. 2015, AJ, 150, 150.

Fortney, J., Marley, M., Laughlin, G., et al. 2016, ApJ, 824, 25.

Fraser, W. \& Kavelaars, J. 2008, Icarus, 198, 452

Fuentes, C. \& Holman, M. 2008, AJ, 136, 83 
Gladman, B., Holman, M., Grav, T., Kavelaars, J., Nicholson, P., Aksnes, K. \& Petit, J. 2002, Icarus, 157, 269.

Gladman, B. \& Chan, C. 2006, ApJ, 643, L135.

Gladman, B., Marsden, B. \& VanLaerhoven, C. 2008, in The Solar System Beyond Neptune, eds. M. Barucci, H. Boehnhardt, D. Cruikshank and A. Morbidelli (Tucson: Univ of Arizona Press), 43-57.

Gladman, B., Kavelaars, J., Petit, J., Ashby, M., Parker, J., Coffey, J., Jones, R., Rousselot, P. \& Mousis, O. 2009, ApJ, 697, L91.

Gomes, R., Fernandez, J., Gallardo, T. and Brunini, A. 2008, in The Solar System Beyond Neptune, eds. M. Barucci, H. Boehnhardt, D. Cruikshank and A. Morbidelli (Tucson: Univ of Arizona Press), 259-273.

Gomes, R. 2011, Icarus, 215, 661.

Gomes, R., Soares, J. \& Brasser, R. 2015, Icarus, 258, 37.

Gomes, R., Deienno, R., \& Morbidelli, A. 2016, arXiv:1607.05111

Gulbis, A., Elliot, J., Adams, E., Benecchi, S., Buie, M., Trilling, D. \& Wasserman, L. 2010, AJ, 140, 350 .

Hees, A., Folkner, W., Jacobson, R. \& Park, R. 2014, PhRvD.89j2002H.

Holman, M. \& Payne, M. 2016a arXiv160309008.

Holman, M. \& Payne, M. 2016b arXiv160403180.

Hsieh, H., Denneau, L., Wainscoat, R. et al. 2015, Icarus, 248, 289.

Iorio, L. 2014, arXiv:1407.5894.

Jacoby, G., Liang, M., Vaughn, D., Reed, R., \& Armandroff, T. 1998, Proc. SPIE, 3355, 721.

Jedicke, R., Larsen, J. \& Spahr, T. 2002, in Asteroids III, eds. W. Bottke et al. (Tucson, Az: Univ. Arizona Press) 71-88

Jewitt, D., Trujillo, C., \& Luu, J. 2000, AJ, 120, 1140

Jewitt, D. 2012, AJ, 143, 66.

Jilkova, L, Portegies Zwart, S., Pijloo, T., \& Hammer, M. 2015, MNRA, 453, 3157.

Kaib, N. and Quinn, T. 2009, Science, 325, 1234.

Kaib, N., Roskar, R., \& Quinn, T. 2011, Icarus, 215, 491.

Kaib, N. \& Sheppard, S. 2016, AJ, submitted. 
Kenyon, S. \& Bromley, B. 2004, Nature, 432, 598.

Kenyon, S. \& Bromely, B. 2015, ApJ, 806, 42.

Kenyon, S. \& Bromely, B. 2016, ApJ, 825, 33.

Kozai, Y. 1962, AJ, 67, 591.

Lawler, S., Shankman, C., Kaib, N., Bannister, M., Gladman, B. \& Kavelaars, J. 2016, arXiv: 160506575 .

Levison, H. \& Duncan, M. 1997, Icarus, 127, 13.

Levison, H., Duncan, M., Brasser, R. \& Kaufmann, D. 2010, Science, 329, 187.

Li, G. \& Adams, F. 2016, ApJ, 823, 3.

Linder, E. \& Mordasini, C. 2016, A\&A, 589, 134.

Luu, J., Marsden, B., Jewitt, D., Trujillo, C., Hergenrother, C., Chen, J. \& Offutt, W. 1997, Nature, 387, 573 .

Madigan, A. \& McCourt, M. 2016, MNRAS, 457, 89.

Malhotra, R., Volk, K. \& Wang, X. 2016, ApJ, 824, 22.

Melita, M., Larwood, D. \& Williams I. 2005, Icarus, 173, 559.

Miyazaki, S., Komiyama, Y., Sekiguchi, M., et al. 2002, PASJ, 54, 833.

Morbidelli, A. \& Levison, H. 2004, AJ, 128, 2564.

Morbidelli, A., Levison, H. and Gomes, R. 2008, in The Solar System Beyond Neptune, eds. M. Barucci, H. Boehnhardt, D. Cruikshank and A. Morbidelli (Tucson: Univ of Arizona Press), 275-292.

Mustill, A., Raymond, S. \& Davies, M. 2016, MNRAS, 460, L109.

Pauco, R, \& Klacka, J. 2016, A\&A, 589, 63.

Petit, J., Kavelaars, J., Gladman, B. et al. 2011, AJ, 142, 131.

Pike, R., Kavelaars, J., Petit, J., Gladman, B., Alexandersen, M., Volk, K. \& Shankman, C. 2015, AJ, 149, 202.

Portegies Zwart, S. \& Jilkova, L. 2015, MNRAS, 451, 144.

Ragozzine, D. \& Brown, M. 2007, AJ, 134, 2160.

Sawyer, D., Daly, P., Howell, S., Hunten, M., \& Schweiker, H. 2010, Proc. SPIE, 7735, 12.

Schwamb, M., Brown, M., Rabinowitz, D. \& Ragozzine, D. 2010, ApJ, 720, 1691.

Schwamb, M., Brown, M. \& Fraser, W. 2014, AJ, 147, 2. 
Sheppard, S. \& Trujillo, C. 2006, Science, 313, 511.

Sheppard, S. \& Trujillo, C. 2009, Icarus, 202, 12.

Sheppard, S. 2010, AJ, 139, 1394.

Sheppard, S. \& Trujillo, C. 2010a, ApJ, 723, L233.

Sheppard, S. \& Trujillo, C. 2010b, Science, 329, 1304.

Sheppard, S., Ragozzine, D. \& Trujillo, C. 2012, AJ, 143, 58.

Sheppard, S. \& Trujillo, C. 2015, AJ, 149, 44.

Sheppard, S., Trujillo, C., \& Tholen, D. 2016, ApJ, 825, L13.

Sivaram, C., Kenath, A., \& Kiren, O. 2016, Ap\&SS, 361, 230.

Snodgrass, C., Carry, B., Dumas, C. \& Hainaut, O. 2010, AA, 511, A72.

Soares, J., and Gomes, R. 2013, AA, 553, 110.

Trujillo, C., Jewitt, D., \& Luu, J. 2001, AJ, 122, 457.

Trujillo, C., Sheppard, S., \& Schaller, E. 2011, ApJ, 730, 105.

Trujillo, C. \& Sheppard, S. 2014, Nature, 507, 471.

Tsiganis, K., Gomes, R., Morbidelli, A., \& Levison, H. 2005, Nature, 435, 459.

Whitmire, D. 2016, MNRAS, 455, 114.

Wolfe, T., Reed, R., Blouke, M., Boroson, T,., Armandroff, T., \& Jacoby, G. 1998, Proc. SPIE $3355,487$. 
Table 1. Inner Oort Cloud Survey Observations

\begin{tabular}{cccc}
\hline \hline $\begin{array}{c}\text { UT Date } \\
\text { yyyy/mm/dd }\end{array}$ & Telescope & $\begin{array}{c}\text { Limit } \\
\left(\mathrm{m}_{r}\right)\end{array}$ & $\begin{array}{c}\text { Area } \\
\left(\text { deg }^{2}\right)\end{array}$ \\
\hline $2007 / 10 / 14$ & Subaru & 25.5 & 5.5 \\
$2007 / 10 / 15$ & Subaru & 24.3 & 1.0 \\
$2007 / 10 / 15$ & Magellan & 24.7 & 1.5 \\
$2007 / 10 / 16$ & Magellan & 23.3 & 1.4 \\
$2008 / 06 / 07$ & Magellan & 24.6 & 4.1 \\
2008/06/08 & Magellan & 24.9 & 2.2 \\
$2009 / 06 / 21$ & Subaru & 25.2 & 3.1 \\
$2009 / 10 / 14$ & Subaru & 25.7 & 8.5 \\
$2010 / 04 / 20$ & Magellan & 25.5 & 1.4 \\
$2010 / 04 / 21$ & Magellan & 25.5 & 1.6 \\
$2010 / 06 / 15$ & Subaru & 25.3 & 3.3 \\
$2010 / 06 / 16$ & Subaru & 25.5 & 1.8 \\
$2011 / 03 / 04$ & Magellan & 25.5 & 3.6 \\
$2011 / 03 / 05$ & Magellan & 25.4 & 3.4 \\
$2011 / 04 / 04$ & Magellan & 25.2 & 1.7 \\
$2011 / 04 / 04$ & Magellan & 25.5 & 1.7 \\
$2011 / 07 / 03$ & Magellan & 25.0 & 3.2 \\
$2011 / 09 / 27$ & Magellan & 25.5 & 1.8 \\
$2011 / 09 / 28$ & Magellan & 25.5 & 2.4 \\
$2012 / 03 / 16$ & KPNO4m & 24.3 & 4.7 \\
$2012 / 03 / 17$ & KPNO4m & 24.6 & 6.4 \\
$2012 / 03 / 23$ & Magellan & 25.6 & 2.3 \\
$2012 / 03 / 24$ & Magellan & 25.6 & 2.9 \\
$2012 / 03 / 25$ & Magellan & 25.6 & 3.7 \\
$2012 / 07 / 21$ & Subaru & 25.5 & 4.5 \\
$2012 / 07 / 22$ & Subaru & 25.7 & 6.6 \\
$2012 / 10 / 12$ & Magellan & 25.3 & 1.9 \\
$2012 / 10 / 16$ & Magellan & 25.4 & 2.4 \\
$2012 / 11 / 04$ & CTIO4m & 24.0 & 10.8 \\
$2012 / 11 / 05$ & CTIO4m & 23.8 & 1.5 \\
& & &
\end{tabular}


Table 1-Continued

\begin{tabular}{|c|c|c|c|}
\hline $\begin{array}{c}\text { UT Date } \\
\text { yyyy } / \mathrm{mm} / \mathrm{dd}\end{array}$ & Telescope & $\begin{array}{l}\text { Limit } \\
\left(\mathrm{m}_{r}\right)\end{array}$ & $\begin{array}{c}\text { Area } \\
\left(\operatorname{deg}^{2}\right)\end{array}$ \\
\hline $2012 / 12 / 11$ & $\mathrm{CTIO} 4 \mathrm{~m}$ & 24.3 & 37.8 \\
\hline 2013/03/10 & Magellan & 25.5 & 3.7 \\
\hline $2013 / 03 / 11$ & Magellan & 25.3 & 3.3 \\
\hline 2013/03/12 & Magellan & 25.3 & 2.8 \\
\hline 2013/03/16 & CTIO4m & 24.6 & 64.8 \\
\hline $2013 / 03 / 17$ & CTIO4m & 24.3 & 54.0 \\
\hline $2013 / 05 / 08$ & CTIO4m & 24.2 & 43.2 \\
\hline 2013/05/09 & CTIO4m & 24.0 & 27.0 \\
\hline 2013/08/11 & Magellan & 25.5 & 1.2 \\
\hline $2013 / 10 / 28$ & Magellan & 25.3 & 2.3 \\
\hline 2013/10/29 & Magellan & 24.8 & 0.7 \\
\hline $2013 / 10 / 30$ & Magellan & 25.0 & 2.3 \\
\hline 2013/11/03 & CTIO4m & 24.0 & 32.4 \\
\hline 2013/11/03 & CTIO4m & 24.2 & 24.3 \\
\hline $2013 / 11 / 04$ & CTIO4m & 24.4 & 59.4 \\
\hline $2013 / 11 / 05$ & CTIO4m & 24.5 & 62.1 \\
\hline $2014 / 03 / 24$ & CTIO4m & 24.8 & 64.8 \\
\hline $2014 / 03 / 25$ & CTIO4m & 24.7 & 54.0 \\
\hline $2014 / 03 / 26$ & CTIO4m & 24.5 & 59.4 \\
\hline $2014 / 03 / 27$ & CTIO4m & 24.5 & 37.8 \\
\hline $2014 / 03 / 28$ & CTIO4m & 24.4 & 64.8 \\
\hline $2014 / 08 / 29$ & Subaru & 25.7 & 6.4 \\
\hline $2014 / 09 / 17$ & $\mathrm{CTIO} 4 \mathrm{~m}$ & 24.0 & 13.5 \\
\hline $2014 / 09 / 18$ & CTIO4m & 24.0 & 29.7 \\
\hline 2014/09/19 & CTIO4m & 24.2 & 29.7 \\
\hline $2014 / 09 / 21$ & CTIO4m & 24.4 & 59.4 \\
\hline $2014 / 09 / 22$ & CTIO4m & 24.6 & 54.0 \\
\hline $2015 / 04 / 13$ & $\mathrm{CTIO} 4 \mathrm{~m}$ & 24.1 & 51.3 \\
\hline $2015 / 04 / 14$ & $\mathrm{CTIO} 4 \mathrm{~m}$ & 24.3 & 32.4 \\
\hline 2015/11/14-15 & Magellan & 25.5 & 0.5 \\
\hline
\end{tabular}


Table 1 - Continued

\begin{tabular}{cccc}
\hline \hline $\begin{array}{c}\text { UT Date } \\
\text { yyyy } / \mathrm{mm} / \mathrm{dd}\end{array}$ & Telescope & $\begin{array}{c}\text { Limit } \\
\left(\mathrm{m}_{r}\right)\end{array}$ & $\begin{array}{c}\text { Area } \\
\left(\mathrm{deg}^{2}\right)\end{array}$ \\
\hline
\end{tabular}

This is an abridged version of Table 1. To see the full Table 1, please view the paper at the Astronomical Journal where Table 1 includes all the field pointings in the survey to date. The instruments used were SuprimeCam on Subaru, IMACS on Magellan, MOSAIC-1.1 on KPNO4m, and DECam on CTIO4m. See the text for descriptions of the instruments and telescopes. The limiting magnitude is the r-band magnitude where we would have found at least $50 \%$ of the slow moving objects in the field. 
Table 2. New Extreme Distant Solar System Objects

\begin{tabular}{|c|c|c|c|c|c|c|c|c|c|c|}
\hline Name & $\begin{array}{c}q \\
(\mathrm{AU})\end{array}$ & $\begin{array}{c}a \\
(\mathrm{AU})\end{array}$ & $e$ & $\begin{array}{c}i \\
(\operatorname{deg})\end{array}$ & $\begin{array}{c}\Omega \\
(\operatorname{deg})\end{array}$ & $\begin{array}{c}\omega \\
(\operatorname{deg})\end{array}$ & $\begin{array}{c}b \\
(\operatorname{deg})\end{array}$ & $\begin{array}{l}\text { Dist } \\
(\mathrm{AU})\end{array}$ & $\begin{array}{c}\text { Dia } \\
(\mathrm{km})\end{array}$ & $\begin{array}{c}m_{r} \\
(\mathrm{mag})\end{array}$ \\
\hline \multicolumn{11}{|c|}{ Inner Oort Cloud } \\
\hline 2012 VP113 & 80.3 & 262 & 0.694 & 24.00 & 90.8 & 292.9 & 16.8 & 82.7 & 550 & 23.35 \\
\hline \multicolumn{11}{|c|}{ Extreme Detached Objects } \\
\hline 2014 SR349 & 48 & 288 & 0.84 & 17.98 & 34.8 & 341.3 & 17.1 & 57.2 & 200 & 24.1 \\
\hline 2013 FT28 & 43.6 & 310 & 0.859 & 17.337 & 217.77 & 40.20 & 7.0 & 58.9 & 200 & 24.2 \\
\hline \multicolumn{11}{|c|}{ Detached/MMR+KR Objects } \\
\hline 2014 SS349 & 45.5 & 142 & 0.68 & 48.31 & 144.22 & 147.8 & 12.8 & 52.9 & 125 & 24.6 \\
\hline \multicolumn{11}{|c|}{ Extreme Scattered Objects } \\
\hline 2013 UH15 & 35.0 & 172 & 0.80 & 26.14 & 176.62 & 283.1 & 12.1 & 52.1 & 125 & 24.8 \\
\hline 2013 FS28 & 34.5 & 196 & 0.83 & 13.01 & 204.67 & 101.5 & 2.0 & 87.9 & 400 & 24.3 \\
\hline \multicolumn{11}{|c|}{ Outer Oort Cloud } \\
\hline 2014 FE72 & 36.3 & 2155 & 0.98 & 20.60 & 336.77 & 134.4 & 11.0 & 59.6 & 250 & 23.7 \\
\hline
\end{tabular}

Quantities are the perihelion $(q)$, semi-major axis $(a)$, eccentricity $(e)$, inclination $(i)$, longitude of the ascending node $(\Omega)$, argument of perihelion $(\omega)$, ecliptic latitude at discovery $(b)$, and distance at discovery (Dist). Diameter (Dia) estimates assume a moderate albedo of 0.10. The definitions of the various groupings are: Inner Oort Cloud $(q>50 \& 150<$ $a<1000 \mathrm{AU})$, Outer Oort Cloud $(q>35 A U \& a>1500 \mathrm{AU})$, Extreme Detached Objects $(40<q<50 \& 150<a<1000 \mathrm{AU})$, Detached/MMR+KR Objects $(q>40 \& 50<a<150$ $\mathrm{AU})$, Extreme Scattered Objects $(30<q<40 \& 150<a<1000 \mathrm{AU})$. Uncertainties are shown by the number of significant digits. 
Table 3. Stability of Extreme Trans-Neptunian Objects

\begin{tabular}{|c|c|c|c|c|c|c|c|c|c|}
\hline Name & $\begin{array}{c}q \\
(\mathrm{AU})\end{array}$ & $\begin{array}{c}a \\
(\mathrm{AU})\end{array}$ & $e$ & $\begin{array}{c}i \\
(\operatorname{deg})\end{array}$ & $\begin{array}{c}\Omega \\
(\mathrm{deg})\end{array}$ & $\begin{array}{c}\omega \\
(\operatorname{deg})\end{array}$ & $\begin{array}{c}\bar{\omega} \\
(\mathrm{deg})\end{array}$ & $\begin{array}{c}N \\
(\mathrm{yr})\end{array}$ & Stable \\
\hline \multicolumn{10}{|c|}{ Inner Oort Cloud } \\
\hline 2012 VP113 & 80.27 & 261 & 0.69 & 24.1 & 90.8 & 292.8 & 23.6 & 3 & Yes \\
\hline Sedna & 76.04 & 499 & 0.85 & 11.9 & 144.5 & 311.5 & 96.0 & 13 & Yes \\
\hline \multicolumn{10}{|c|}{ Extreme Detached } \\
\hline 2010 GB174 & 48.76 & 370 & 0.87 & 21.5 & 130.6 & 347.8 & 118.4 & 4 & Yes \\
\hline 2014 SR349 & 47.6 & 288 & 0.84 & 18.0 & 34.8 & 341.3 & 16.1 & 1 & Yes \\
\hline 2004 VN112 & 47.32 & 318 & 0.85 & 25.6 & 66.0 & 327.1 & 33.1 & 6 & Yes \\
\hline 2000 CR105 & 44.29 & 226 & 0.80 & 22.7 & 128.3 & 317.2 & 85.5 & 6 & Yes:MMR? \\
\hline 2013 FT28 & 43.6 & 310 & 0.86 & 17.3 & 217.8 & 40.2 & 258.0 & 3 & Yes:Res? \\
\hline 2013 GP136 & 41.11 & 153 & 0.73 & 33.5 & 210.7 & 42.2 & 252.9 & 3 & Yes \\
\hline \multicolumn{10}{|c|}{ Extreme Scattered } \\
\hline 2005 RH52 & 38.98 & 151 & 0.74 & 20.5 & 306.1 & 32.3 & 338.4 & 5 & Yes:MMR? \\
\hline 2003 HB57 & 38.10 & 165 & 0.77 & 15.5 & 197.8 & 10.9 & 208.7 & 4 & No \\
\hline 2013 RF98 & 36 & 325 & 0.88 & 29.6 & 67.6 & 316.5 & 24.1 & 0 & No \\
\hline 2007 TG422 & 35.57 & 482 & 0.93 & 18.6 & 112.9 & 285.8 & 38.7 & 4 & No \\
\hline 2002 GB32 & 35.35 & 218 & 0.84 & 14.2 & 177.0 & 37.0 & 214.0 & 5 & No \\
\hline 2007 VJ305 & 35.18 & 188 & 0.81 & 12.0 & 24.4 & 338.3 & 2.7 & 3 & No \\
\hline 2013 UH15 & 35.0 & 174 & 0.79 & 26.1 & 176.5 & 282.9 & 99.4 & 2 & No \\
\hline 2010 VZ98 & 34.32 & 152 & 0.77 & 4.5 & 117.4 & 313.9 & 71.3 & 6 & No \\
\hline 2001 FP185 & 34.26 & 227 & 0.85 & 30.8 & 179.3 & 7.0 & 186.3 & 8 & No \\
\hline 2013 FS28 & 34.1 & 199 & 0.83 & 13.1 & 204.6 & 102.0 & 306.6 & 1 & No \\
\hline $2015 \mathrm{SO} 20$ & 33.16 & 162 & 0.80 & 23.4 & 33.6 & 354.9 & 28.5 & 4 & No \\
\hline
\end{tabular}

Orbits are from the Minor Planet Center (MPC) as of July 2016. Bolded extreme objects are in the anti $180 \mathrm{deg}$ secondary longitude of perihelion group and show opposite argument of perihelia and orbit pole angles than the main group of clustered objects. Quantities are the perihelion $(q)$, semi-major axis $(a)$, eccentricity $(e)$, inclination $(i)$, longitude of the ascending node $(\Omega)$, argument of perihelion $(\omega)$, longitude of perihelion $(\bar{\omega})$, and Number of years observed. An object is considered stable if it moved less than a few AU in semimajor axis during our 1 billion year numerical integrations. 2000 CR105 and 2005 RH52 showed stable semi-major axes, but the stepped motion of $a$ over time suggests they could 
be in a mean motion resonance with Neptune. 2013 FT28 also had some jumpiness in its semi-major axis, usually about $10 \mathrm{AU}$ for the nominal and 1 sigma clones orbits, suggesting some significant interactions with Neptune, see text. All unstable objects had well over 100 AU changes in their semi-major axes within 1 billion years, with most lost within about 1 billion years including 2010 VZ98 and 2007 TG422. 
Table 4. Other New Interesting Distant Solar System Objects

\begin{tabular}{|c|c|c|c|c|c|c|c|c|c|c|}
\hline Name & $\begin{array}{c}q \\
(\mathrm{AU})\end{array}$ & $\begin{array}{c}a \\
(\mathrm{AU})\end{array}$ & $e$ & $\begin{array}{c}i \\
(\operatorname{deg})\end{array}$ & $\begin{array}{c}\Omega \\
(\operatorname{deg})\end{array}$ & $\begin{array}{c}\omega \\
(\operatorname{deg})\end{array}$ & $\begin{array}{l}\text { Dist } \\
(\mathrm{AU})\end{array}$ & $\begin{array}{c}\text { Dia } \\
(\mathrm{km})\end{array}$ & $\begin{array}{c}m_{r} \\
(\mathrm{mag})\end{array}$ & $N: N$ \\
\hline 2014 SW349 & 39 & 96 & 0.59 & 15.2 & 19.8 & 251.7 & 50.1 & 100 & 24.5 & $17: 3$ \\
\hline 2014 FJ72 & 38 & 94 & 0.59 & 15.4 & 302.7 & 132.7 & 69.3 & 300 & 23.9 & $11: 2 / 17: 3$ \\
\hline 2014 FL72 & 38 & 103 & 0.63 & 29.17 & 201.14 & 258.99 & 62.4 & 175 & 24.6 & $6: 1 / 13: 2$ \\
\hline 2014 SZ349 & 37 & 79 & 0.54 & 35.8 & 152.9 & 135.6 & 50.9 & 125 & 24.7 & 13:3 \\
\hline 2014 SD350 & 34 & 79 & 0.57 & 32.8 & 356.6 & 46.6 & 48.2 & 150 & 24.0 & $13: 3$ \\
\hline
\end{tabular}

Quantities are the perihelion $(q)$, semi-major axis $(a)$, eccentricity $(e)$, inclination $(i)$, longitude of the ascending node $(\Omega)$, argument of perihelion $(\omega)$, distance at discovery (Dist), and location near Neptune resonance $(N: N)$. Diameter (Dia) estimates assume a moderate albedo of 0.10. The uncertainty of the orbital elements for each object are shown by the number of significant digits. 


\begin{tabular}{|c|c|c|}
\hline \multicolumn{3}{|c|}{ Common Parameters } \\
\hline Parameter & Value & Description \\
\hline$\rho$ & $1000 \mathrm{~kg} \mathrm{~m}^{-3}$ & Density \\
\hline$p_{r}$ & 0.10 & Albedo in $r$ filter \\
\hline$r_{\min }$ & $75 \mathrm{~km}$ & Minimum radius \\
\hline$r_{\max }$ & $1200 \mathrm{~km}$ & Maximum radius \\
\hline$a_{\max }$ & $800 \mathrm{AU}$ & Maximum semi-major axis \\
\hline$e_{\min }$ & 0.625 & Minimum eccentricity (uniform distribution) \\
\hline$q^{\prime}$ & 4 or 5 & Size distribution power law exponent \\
\hline$a$ & 1 & Semi-major axis distribution power law exponent \\
\hline$\sigma_{i}$ & $6.9^{\circ}$ & Sigma for Gaussian inclination distribution \\
\hline$\mu$ & $19.1^{\circ}$ & Mean for Gaussian inclination distribution \\
\hline$\omega_{\min }$ & $-80 \mathrm{deg}$ & Minimum argument of perihelion \\
\hline$\omega_{\max }$ & $45 \mathrm{deg}$ & Maximum argument of perihelion \\
\hline \multicolumn{3}{|c|}{ Inner Oort Cloud Parameters } \\
\hline$q_{\min }$ & $75 \mathrm{AU}$ & Minimum perihelion \\
\hline$a_{\min }$ & $200 \mathrm{AU}$ & Minimum semi-major axis \\
\hline $\bar{\omega}_{\min }$ & $0 \mathrm{deg}$ & Minimum longitude of perihelion \\
\hline $\bar{\omega}_{\max }$ & $130 \mathrm{deg}$ & Maximum longitude of perihelion \\
\hline$N_{\text {obs }}$ & 1 & Observed objects: $2012 \mathrm{VP}_{113}$ \\
\hline$N\left(q^{\prime}=4\right)$ & $9.4 \times 10^{3}$ & Best-fit number of objects $r>100 \mathrm{~km}$ \\
\hline$N\left(q^{\prime}=5\right)$ & $2.1 \times 10^{4}$ & Best-fit number of objects $r>100 \mathrm{~km}$ \\
\hline \multicolumn{3}{|c|}{ Extreme Detached Objects and Extreme Scattered Objects ( $\bar{\omega}$ Constrained) } \\
\hline$q_{\min }$ & $35 \mathrm{AU}$ & Minimum perihelion \\
\hline$q_{\max }$ & $50 \mathrm{AU}$ & Maximum perihelion \\
\hline$a_{\min }$ & $150 \mathrm{AU}$ & Minimum semi-major axis \\
\hline $\bar{\omega}_{\min }$ & $0 \mathrm{deg}$ & Minimum longitude of perihelion \\
\hline $\bar{\omega}_{\max }$ & $130 \mathrm{deg}$ & Maximum longitude of perihelion \\
\hline$N_{\text {obs }}$ & 2 & Observed objects: c198c45, M1670c5 \\
\hline$N\left(q^{\prime}=4\right)$ & 1900 & Best-fit number of objects $r>100 \mathrm{~km}$ \\
\hline$N\left(q^{\prime}=5\right)$ & 1800 & Best-fit number of objects $r>100 \mathrm{~km}$ \\
\hline \multicolumn{3}{|c|}{ Extreme Detached Objects and Extreme Scattered Objects ( $\bar{\omega}$ Unconstrained $)$} \\
\hline$q_{\min }$ & $35 \mathrm{AU}$ & Minimum perihelion \\
\hline$q_{\max }$ & $50 \mathrm{AU}$ & Maximum perihelion \\
\hline$a_{\min }$ & $150 \mathrm{AU}$ & Minimum semi-major axis \\
\hline $\bar{\omega}_{\min }$ & $0 \mathrm{deg}$ & Minimum longitude of perihelion \\
\hline $\bar{\omega}_{\max }$ & $360 \mathrm{deg}$ & Maximum longitude of perihelion \\
\hline$N_{\text {obs }}$ & 3 & Observed objects: c198c45, D292c54, M1670c5 \\
\hline$N\left(q^{\prime}=4\right)$ & 2900 & Best-fit number of objects $r>100 \mathrm{~km}$ \\
\hline$N\left(q^{\prime}=5\right)$ & 2700 & Best-fit number of objects $r>100 \mathrm{~km}$ \\
\hline
\end{tabular}

Table 5: Population parameters used for the observational bias simulations. The actual number of simulated objects was at least a factor 10 greater than the best fit number of detected simulated objects in all cases. We report the number of objects for radii greater than $100 \mathrm{~km}$ for the various simulation parameters. Radially asymmetric and symmetric populations (uniform in $\omega$ and $\bar{\omega}$ ) were considered for all populations. 


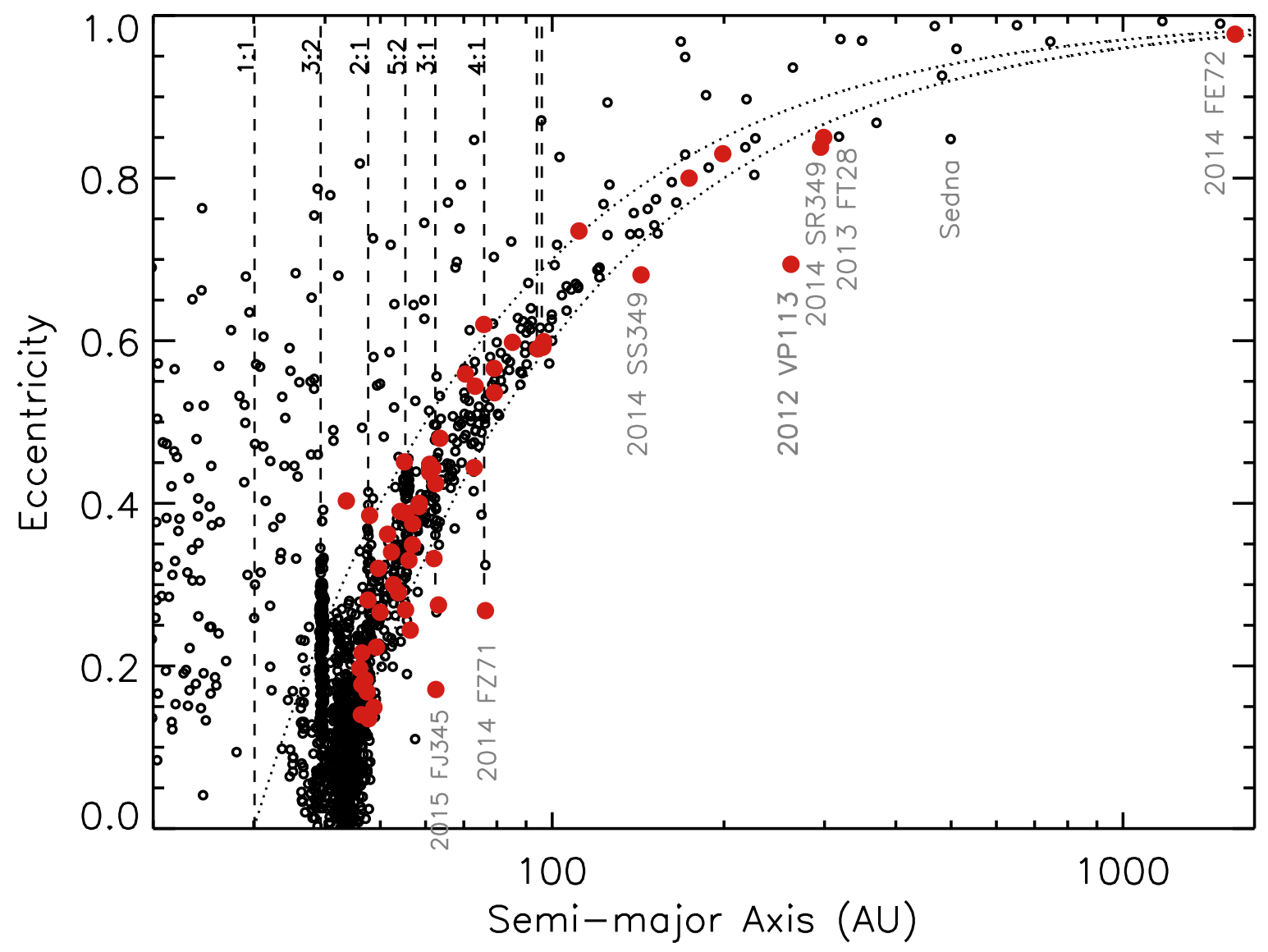

Fig. 1.- The semi-major axis versus eccentricity for all objects with well determined orbits as of July 2016 from the Minor Planet Center. Red circles show the new objects discovered in this survey with well determined orbits. Dashed lines show strong mean motion resonances with Neptune and dotted lines show constant perihelia of 30 and 40 AU. All objects uncertainties are generally smaller than the symbols. 


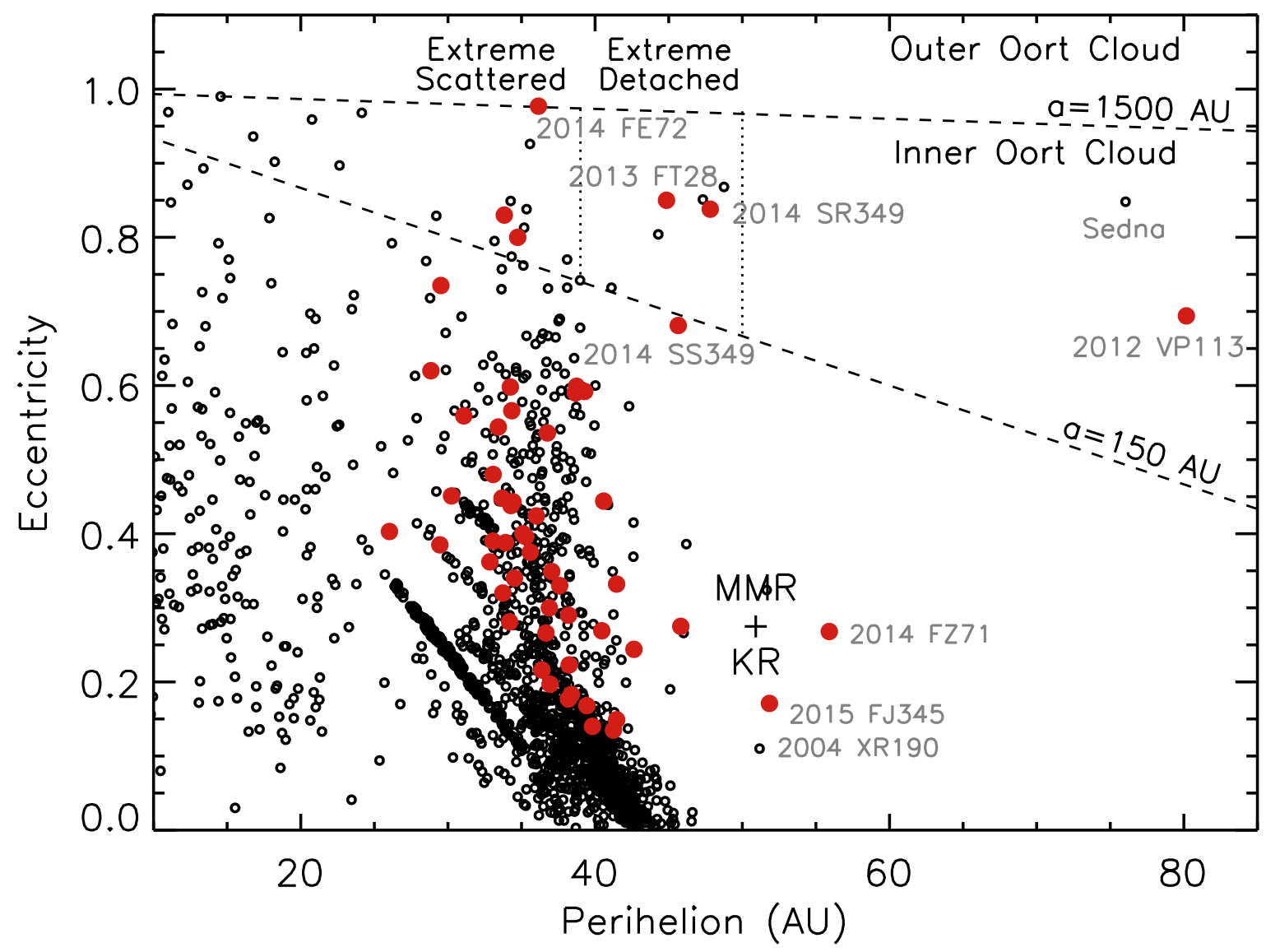

Fig. 2.- The perihelion versus eccentricity of small outer solar system objects with well known orbits as of July 2016 from the Minor Planet Center. Red filled circles are objects discovered during this survey with well determined orbits. Objects above the 150 AU semimajor axis dashed line are considered extreme. Bonafide Inner Oort Cloud objects are considered to have perihelion above $50 \mathrm{AU}$. Extreme detached objects are mostly decoupled from the giant planets and have perihelion between about 40 and $50 \mathrm{AU}$ and may have a similar origin as the IOC objects. Extreme scattered disk objects have perihelia below 40 AU and can have significant interactions with Neptune. Outer Oort cloud objects have semimajor axes above $1500 \mathrm{AU}$ and can have significant interactions with outside forces such as the galactic and stellar tides. Objects with relatively high perihelion beyond the Kuiper Belt edge at $50 \mathrm{AU}$ but only moderate eccentricity are likely created by a combination of past Neptune Mean Motion Resonances (MMR) and the Kozai Resonance (KR) and are detailed in Sheppard et al. (2016). All objects uncertainties are generally smaller than the symbols. 


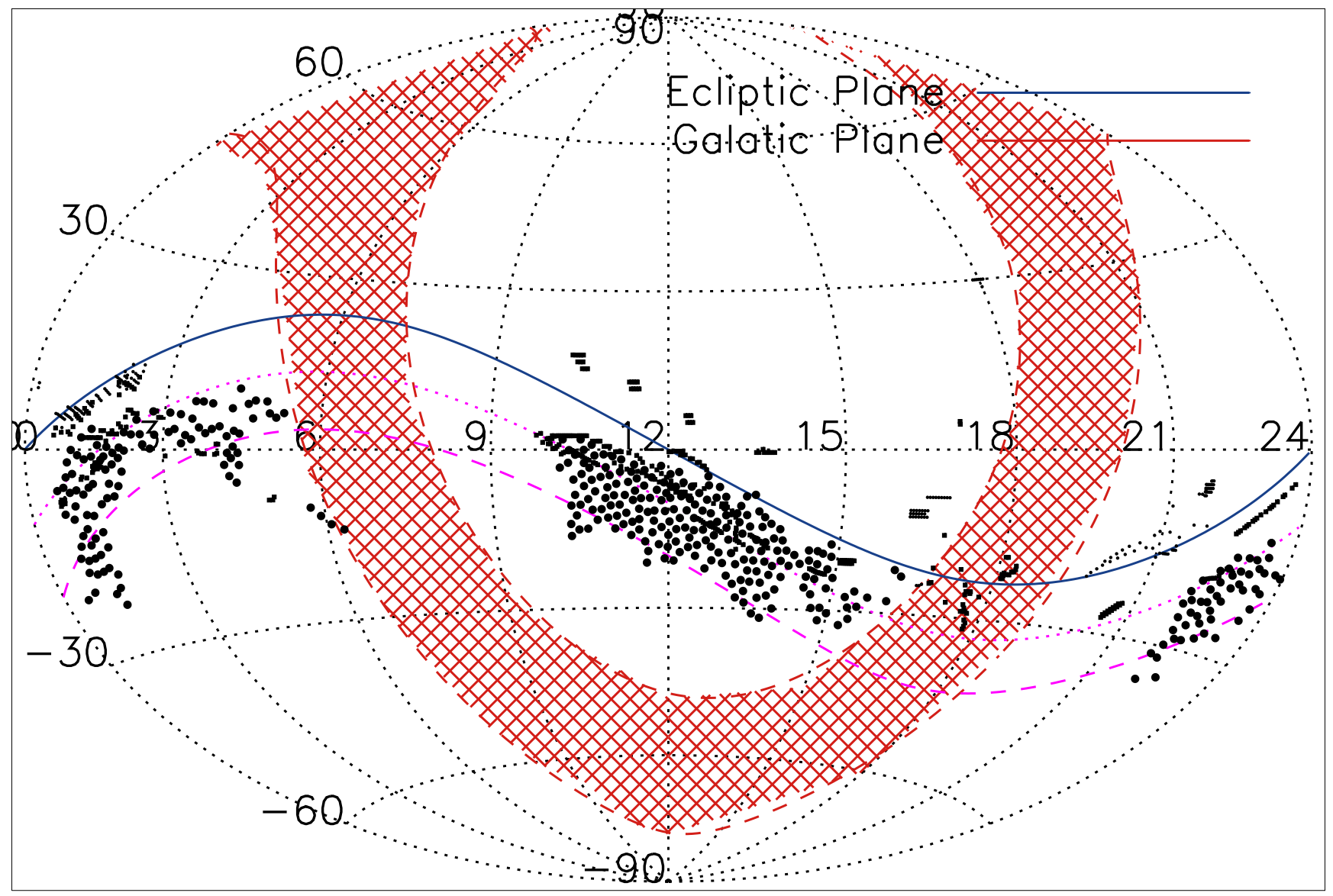

Fig. 3.- The survey fields obtained using the Dark Energy Camera on the CTIO 4m (large circles), MOSAIC2 on the KPNO 4m (large squares), IMACS on Magellan (small circles) and SuprimeCam on Subaru (small squares) as detailed in Table 1. The dotted and dashed purple lines are respectively 10 and 20 degrees South of the ecliptic. The galactic plane is shown in red to \pm 15 degrees of the center. In total, we covered about 1080 square degrees during the survey with an average of 13 square degrees from the ecliptic. 


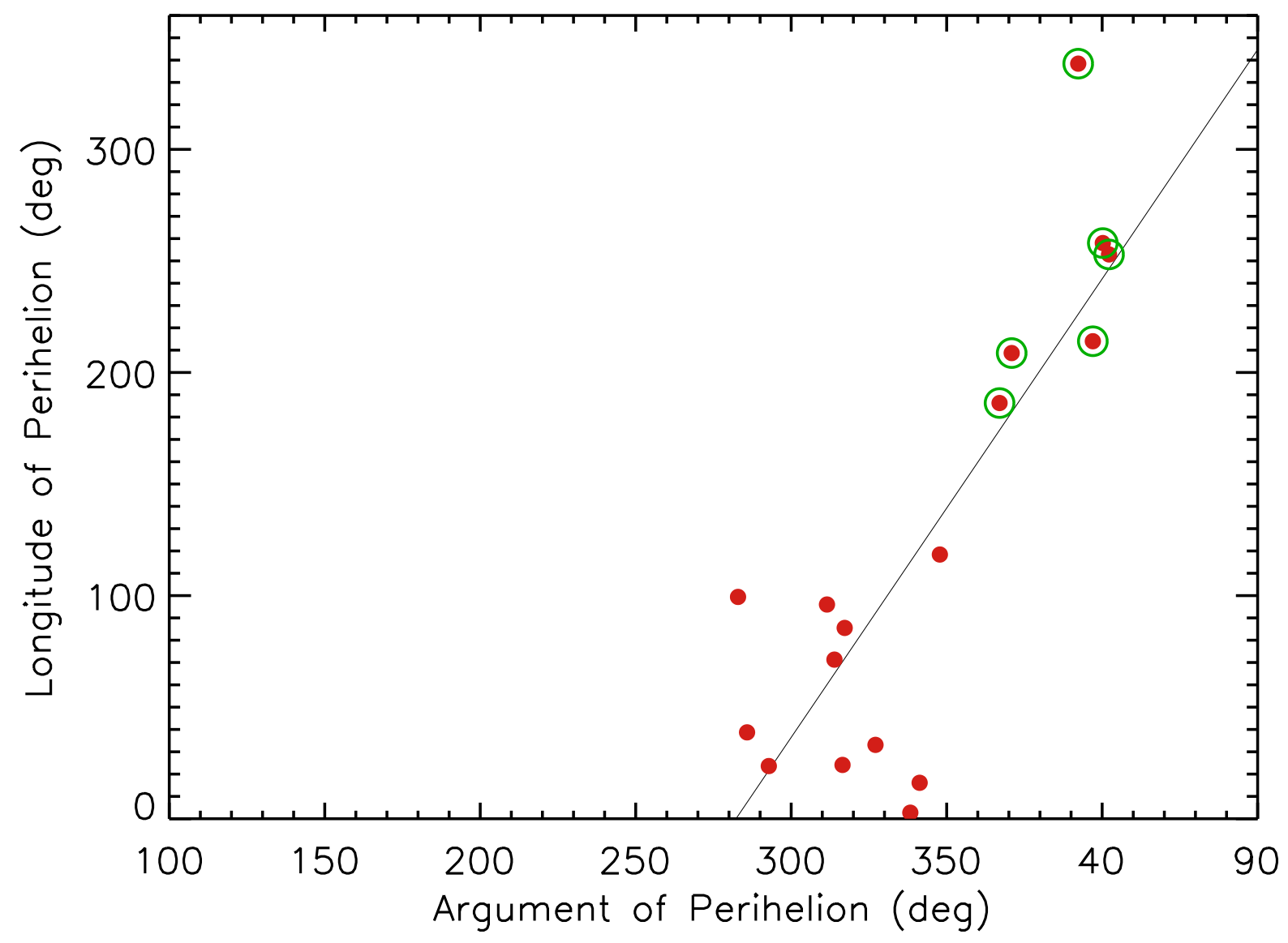

Fig. 4.- Argument of perihelion versus longitude of perihelion for extremely distant transNeptunian objects. There is a clear correlation between these two angles at the $99.99 \%$ level. As explained in the text, this is further evidence of a massive inclined planet at a few hundred AU. Green circles show the objects in the secondary anti-longitude of perihelion cluster. 


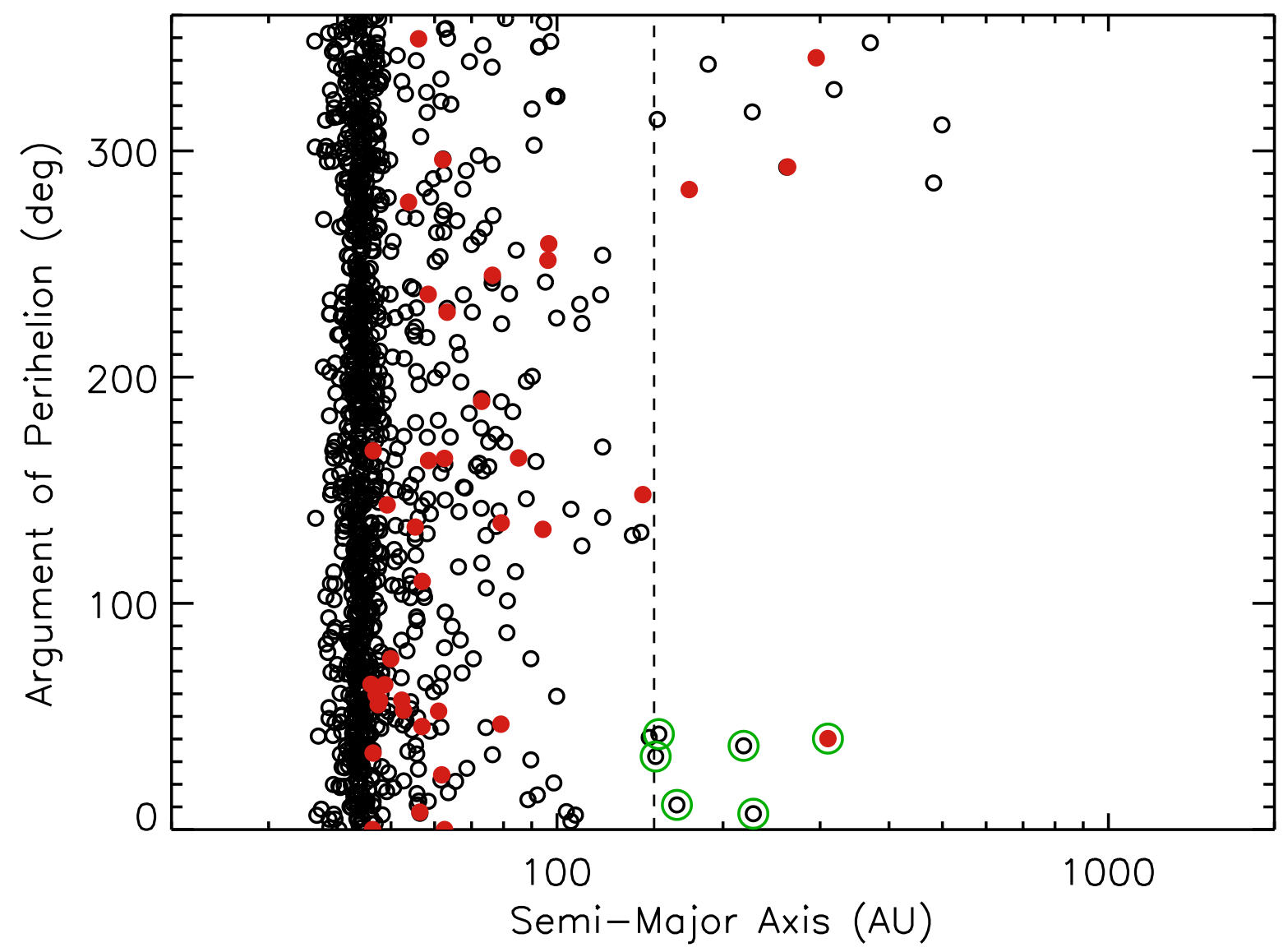

Fig. 5.- Semi-major axis versus argument of perihelion for all objects with perihelia greater than 35 AU. There is a noticeable clustering between 290 and 40 degrees. Red symbols are new objects discovered in this survey. Green circles show the objects that are in the secondary anti-longitude of perihelion clustering group. 


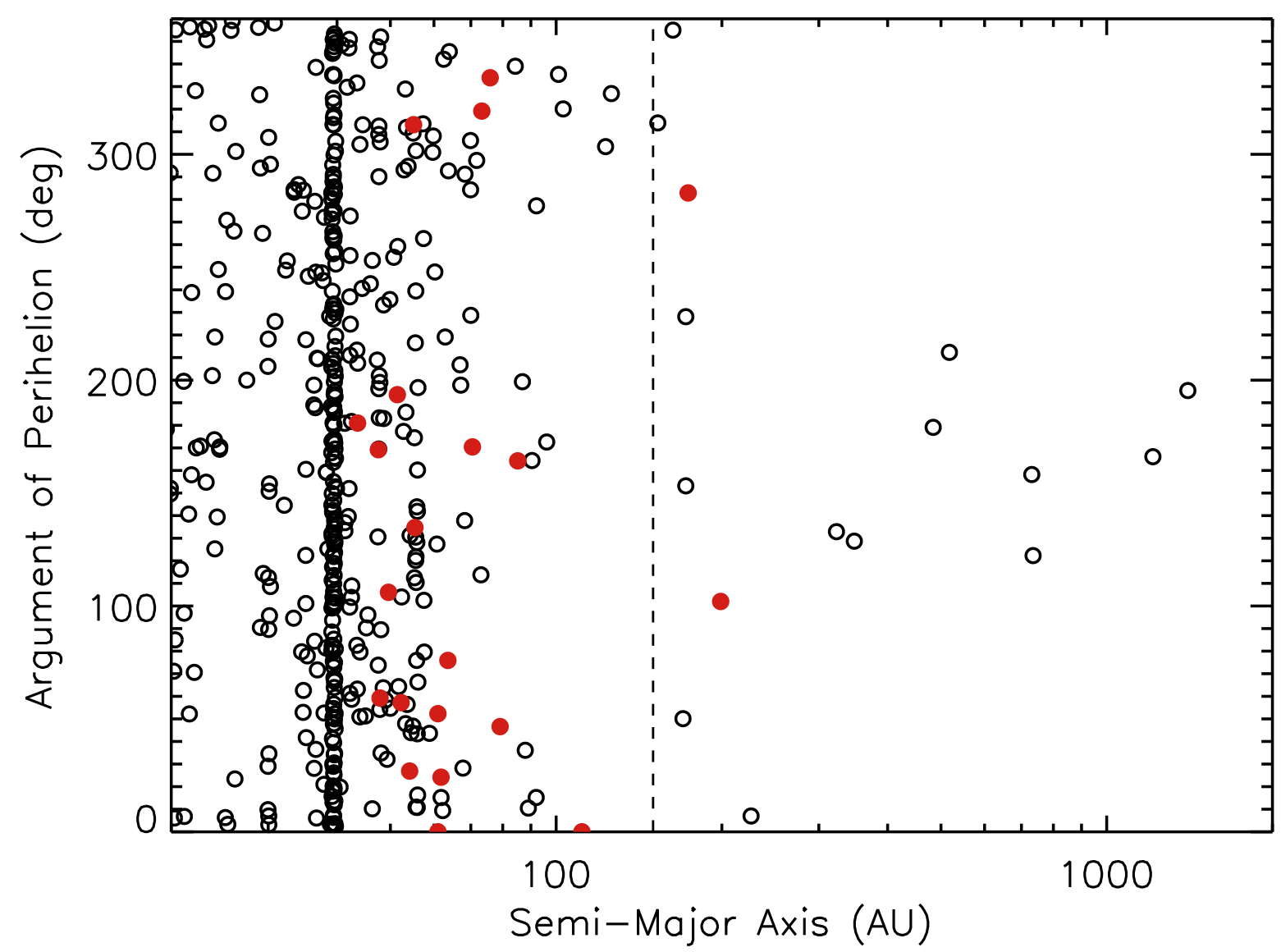

Fig. 6. - Semi-major axis versus argument of perihelion for all objects with perihelia less than 35 AU. There is a noticeable clustering between 100 and 200 degrees, which is the opposite of Figure 5. 


$$
1
$$




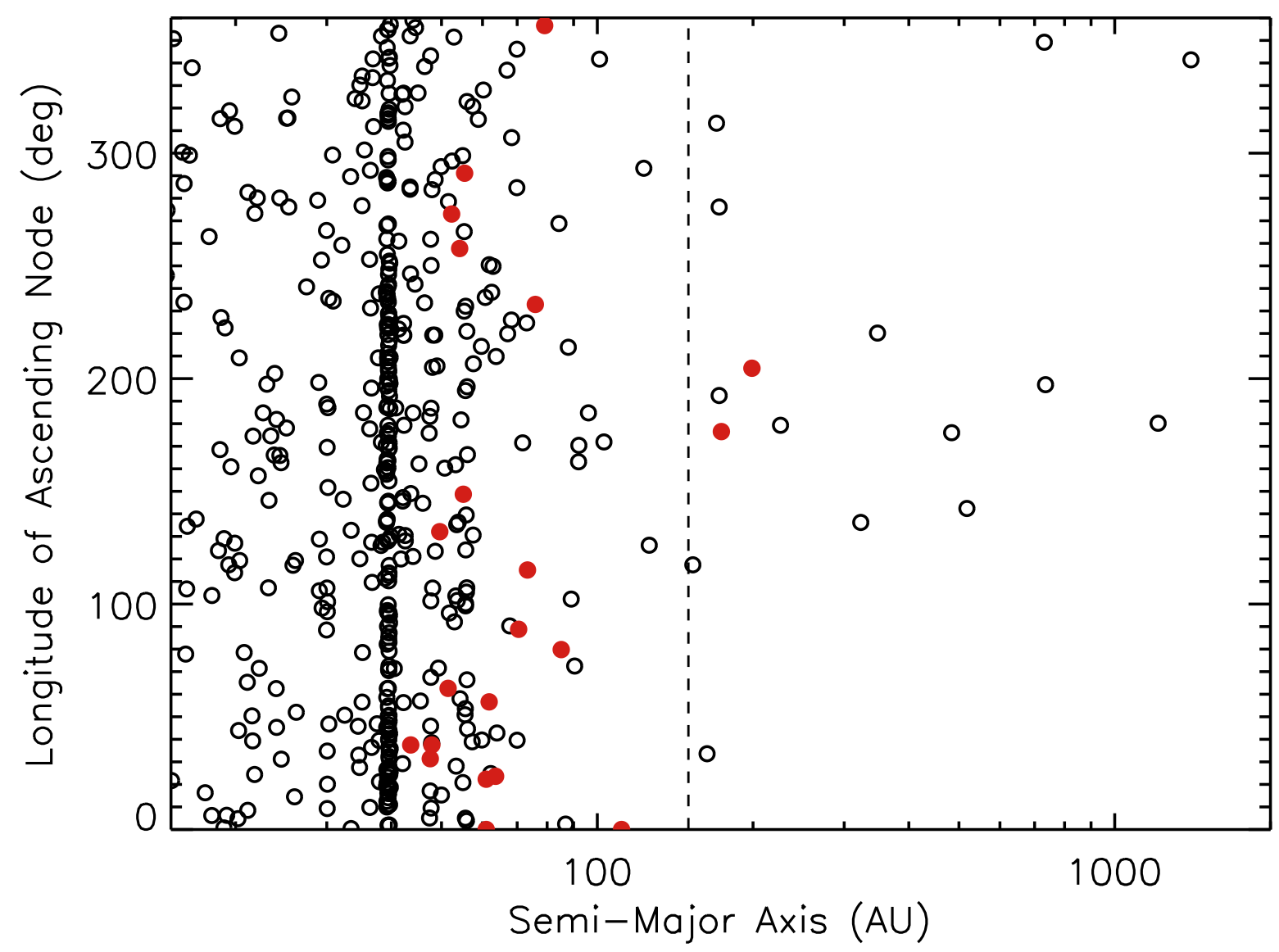

Fig. 8.- Semi-major axis versus longitude of the ascending node with perihelia less than 35 AU. There is a clustering near 180 degrees. 


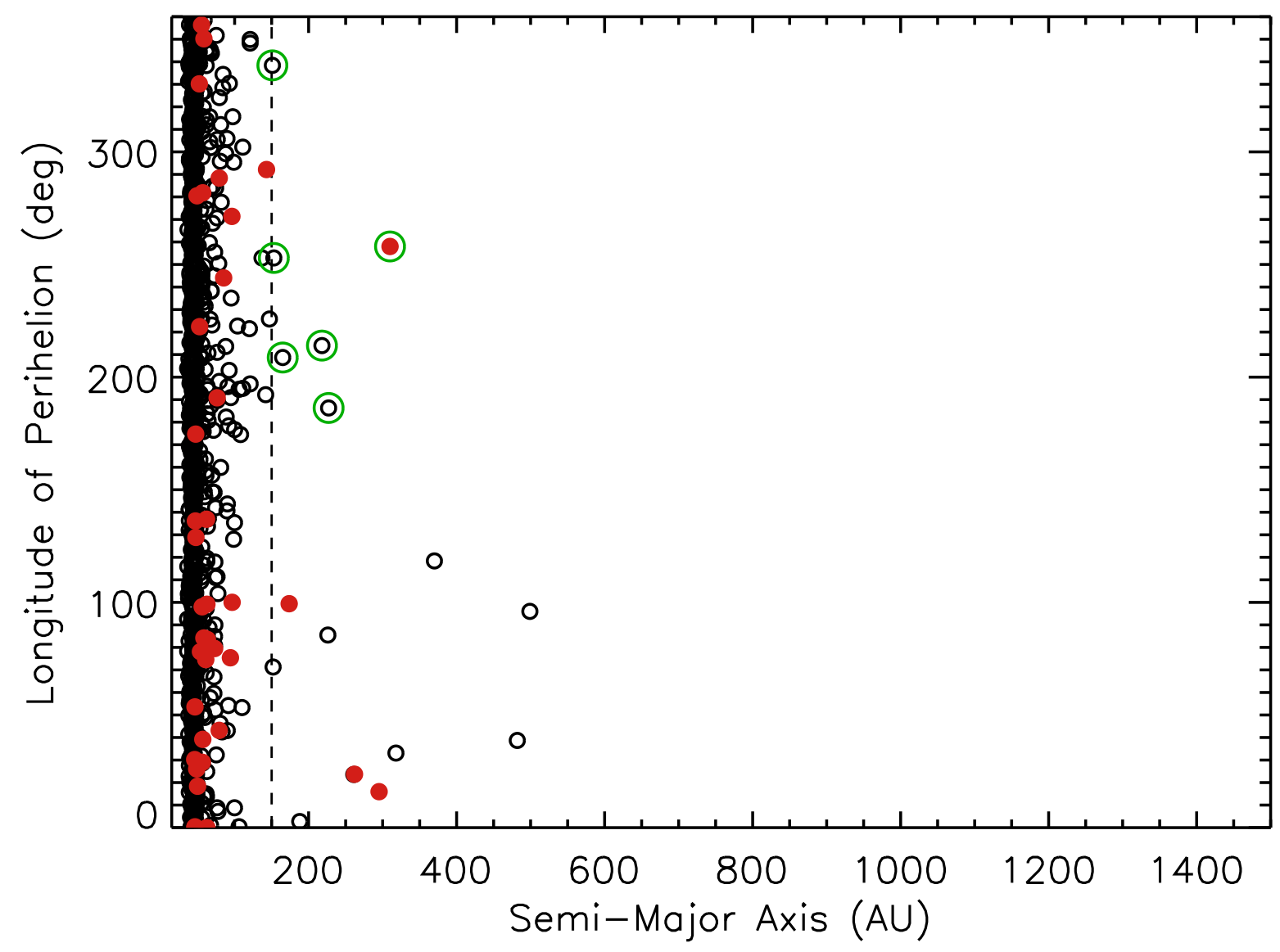

Fig. 9.- Semi-major axis versus longitude of perihelion with perihelia greater than 35 AU. There is a main clustering between 0 and 130 degrees for most extreme objects with a possible second clustering for lower semi-major axes between 200 and 260 degrees (circled in green). 2013 FT28 does not follow the longitude of perihelion clustering of the other extreme TNOs with the highest semi-major axes, but may fall into the secondary clustering region. Red dots are new discoveries from this survey. 


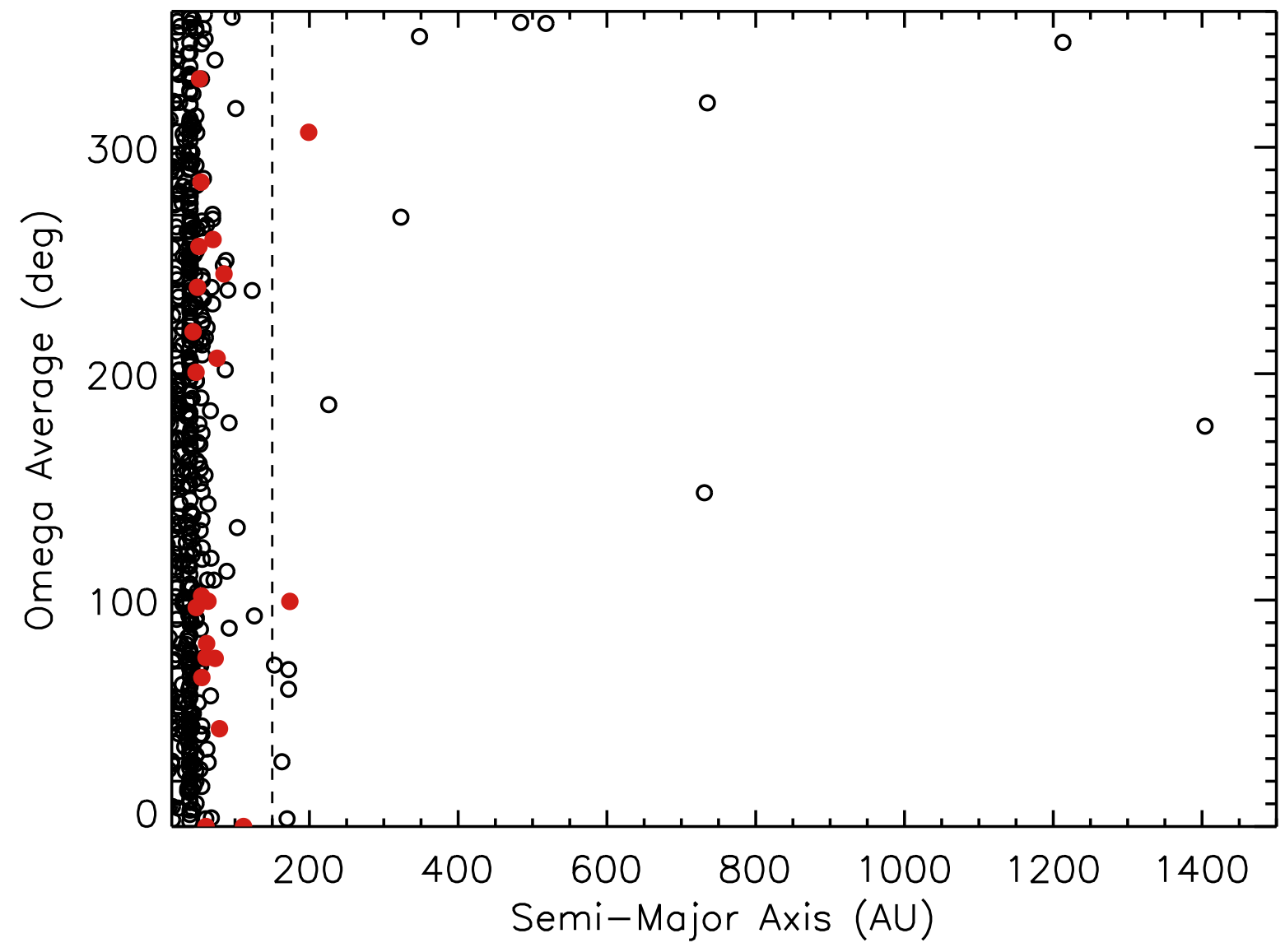

Fig. 10.- Semi-major axis versus longitude of perihelion with perihelia less than 35 AU. There are marginal clusterings near 0 degrees and 180 degrees. 


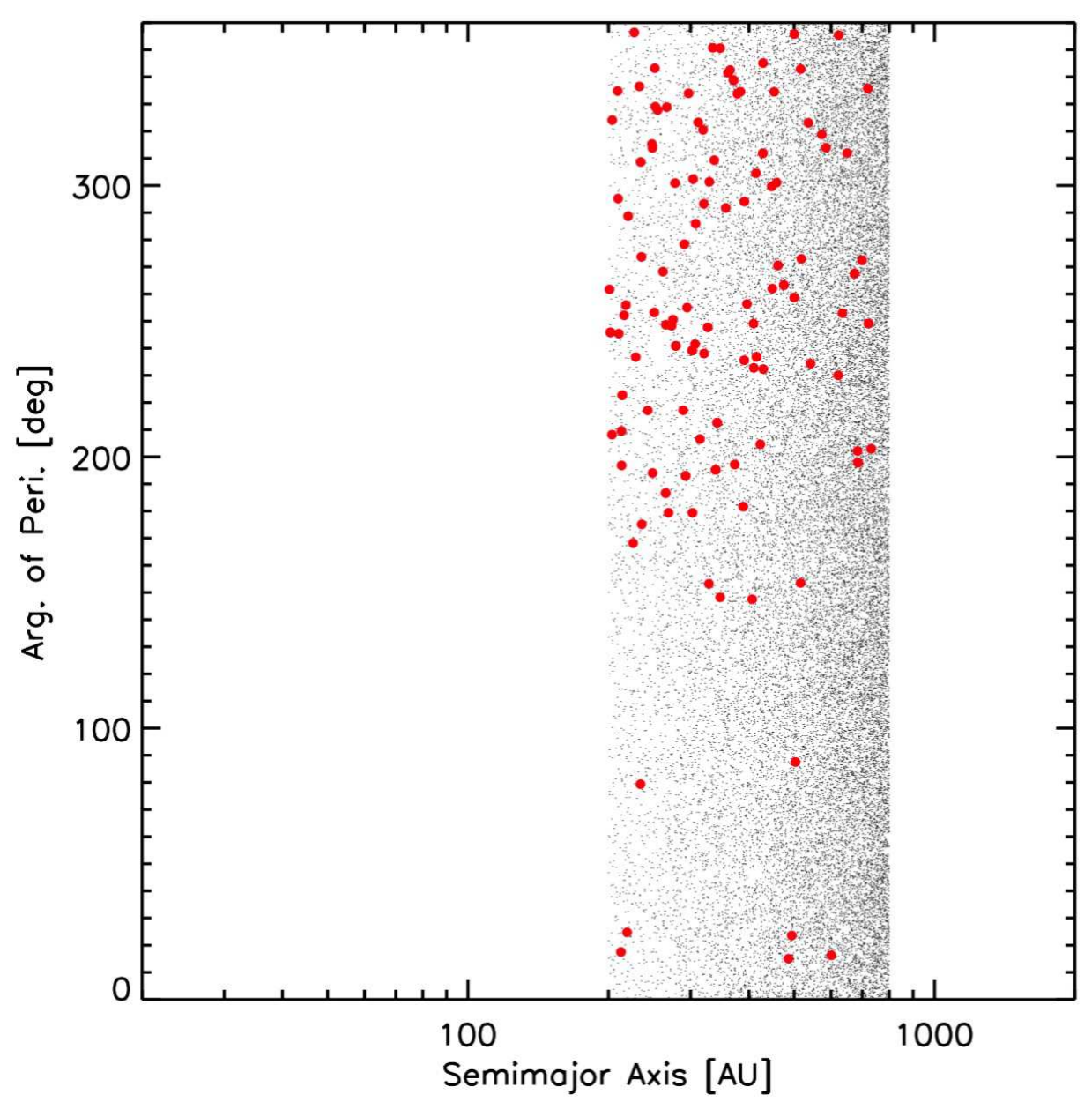

Fig. 11. - Semi-major axis versus argument of perihelion for a symmetric ETNO distribution. Small black dots are simulated objects and larger red dots are objects that would have been detected in our survey given our sky coverage. Because most of our survey was done South of the ecliptic, we are less sensitive to detecting objects with arguments of perihelion between 0 and 180 degrees. Despite this secondary bias, as explained in the text, we still find strong evidence for the argument of perihelion clustering for ETNOs near 0 degrees where all are known as we are still just as likely to find ETNOs near 180 degrees, where none are known. 


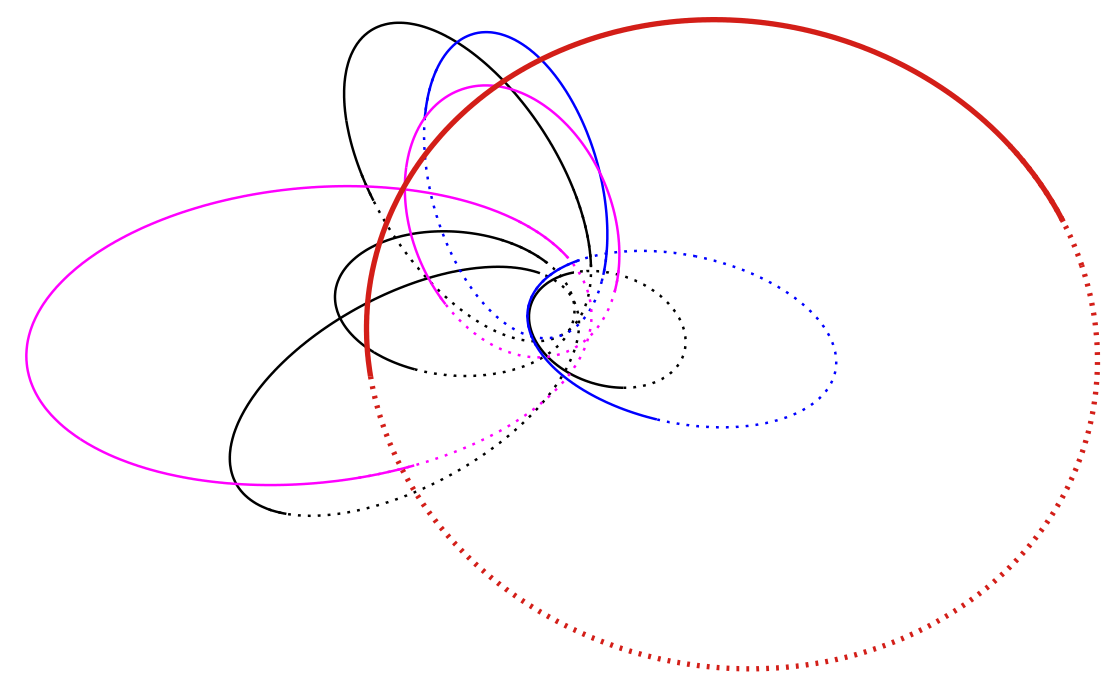

Fig. 12.- A top down view of the orbits of the extreme detached trans-Neptunian objects (black) and Inner Oort Cloud objects (purple) with $a>150$ and $q>40 \mathrm{AU}$. The new extreme detached objects discovered in this survey are shown in blue (2014 SR349 and 2013 FT28). The BB2016 planet orbit prediction is shown in red. The dashed part of an orbit is when the object is below the ecliptic. The new object 2013 FT28 does not fit the longitude of perihelion trend shown by the other EDTNOs and IOC objects, but its location nearly 180 degrees away suggests a secondary anti clustering in longitude of perihelion for ETNOs. We find a distinct $180 \mathrm{deg}$ anti group amongst the ETNOs that also has opposite argument of perihelia and orbit pole angles from the main clustering. The two ETNO groups seem to show some sort of resonance behaviour with multiple orbital angles set to keep them away from the planet. Though we only plot the stable ETNOs here for clarity, all ETNOs from Table 3, stable and unstable, in both clusters show similar behaviour befitting the cluster group. 


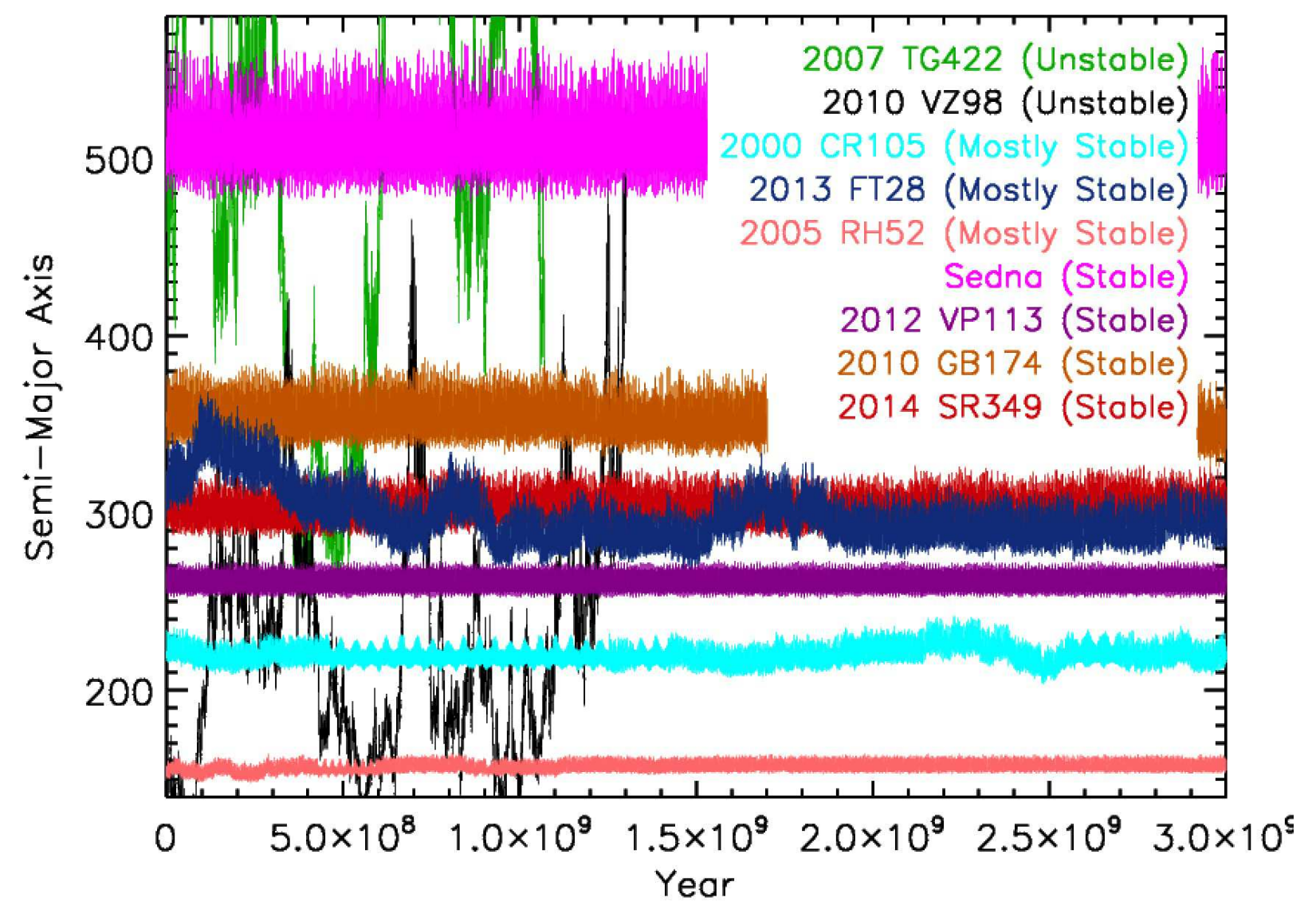

Fig. 13. - The numerically integrated nominal orbits of 2013 FT28, 2014 SR349, 2012 VP113, 2010 VZ98, 2010 GB174, 2007 TG422, 2005 RH52, 2000 CR105 and Sedna. 2014 SR349, like Sedna, 2012 VP113, and 2010 GB174 is very stable over the age of the solar system. 2007 TG422 and 2010 VZ98 appear to be very unstable and are lost within about 1 billion years. 2013 FT28 is mostly stable, but shows some significant semi-major axis jumps suggesting resonant dynamical interactions with the giant planets. 2000 CR105 also is mostly stable but shows small semi-major axis spikes indicative of resonance interactions with the giant planets. 2005 RH52 also shows some semi-major axis spikes. See Table 3 for a full list of ETNO orbit stability results. It is clear the larger the semi-major axis of the stable extreme objects, the larger the range in semi-major axis the object has over the age of the solar system (a few AU for 2005 RH52 near 150 AU and around 50 AU for Sedna near $500 \mathrm{AU})$. 


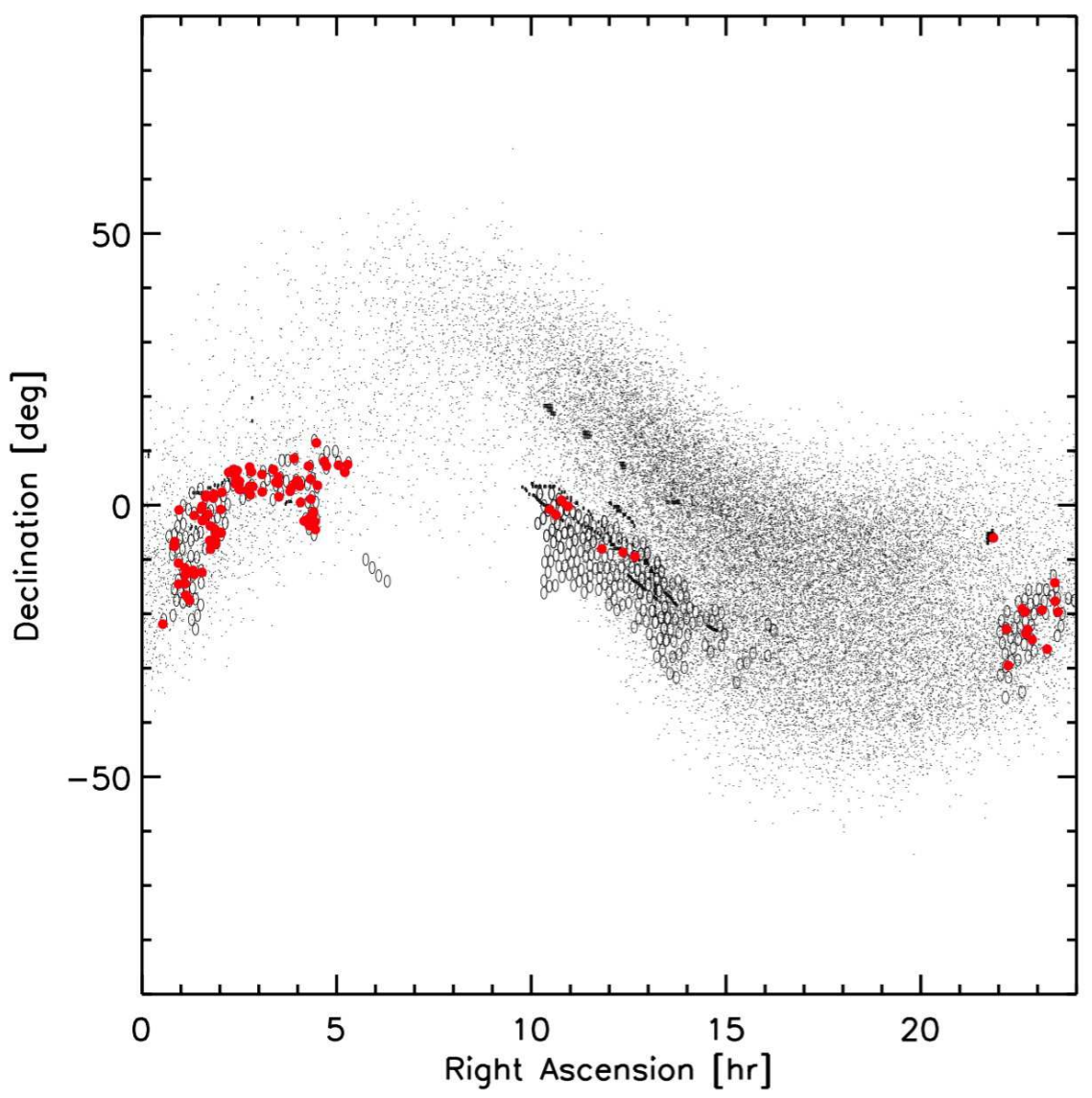

Fig. 14.- Our survey fields (open circles) shown with simulated discovered objects (red) from our survey simulator. Only a fraction of the objects in our simulation are shown as small black dots. This simulation was based on the extreme trans-Neptunian objects as having an asymmetric orbital distribution where their longitudes of perihelion are all near Sedna and 2012 VP113. Thus most of the objects would be located near aphelion around 14 to 19 hours in right ascension while most discoveries would be made when the objects are brightest near perihelion between 2 and 7 hours. This figure shows the bias a survey would have in discovering extreme objects if only obtained near one particular longitude. 


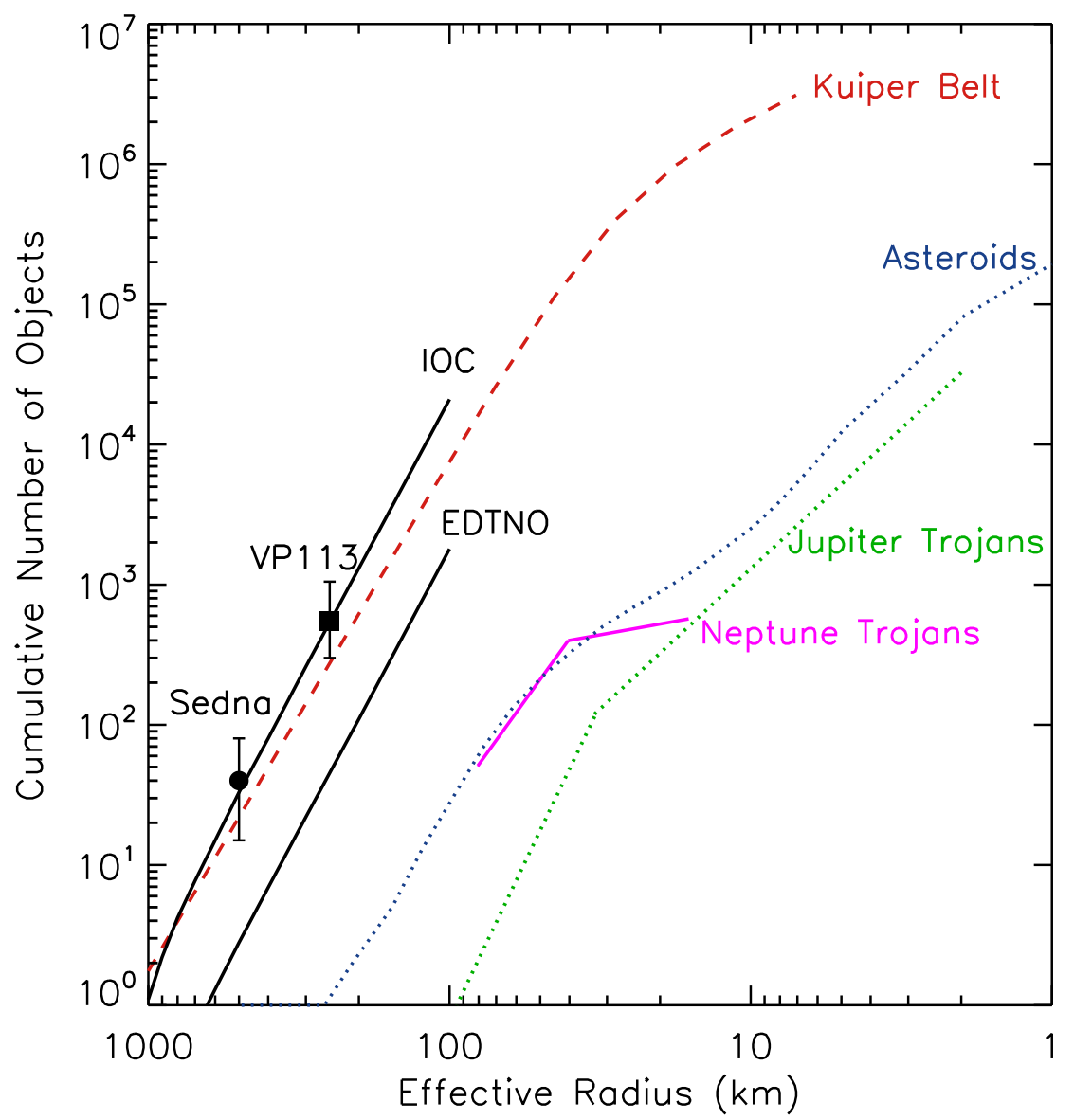

Fig. 15. - The cumulative number of objects in the known stable reservoirs in our solar system. The IOC population $(q>50 \mathrm{AU})$ is likely the largest observed reservoir of small objects in our solar system and should have a few objects larger than Pluto within the population. The errors on the population are shown by the 2012 VP113 and Sedna locations. The extreme detached trans-Neptunian objects $(40<q<50$ AU) are likely smaller than the IOC population. Both the IOC and EDTNO population estimates are based on our survey and is explained in the text. (Population estimates are from This Work, Jewitt et al. 2000; Jedicke et al. 2002; Bottke et al. 2005; Fraser \& Kavelaars 2008; Fuentes \& Holman 2008; Sheppard \& Trujillo 2010). 


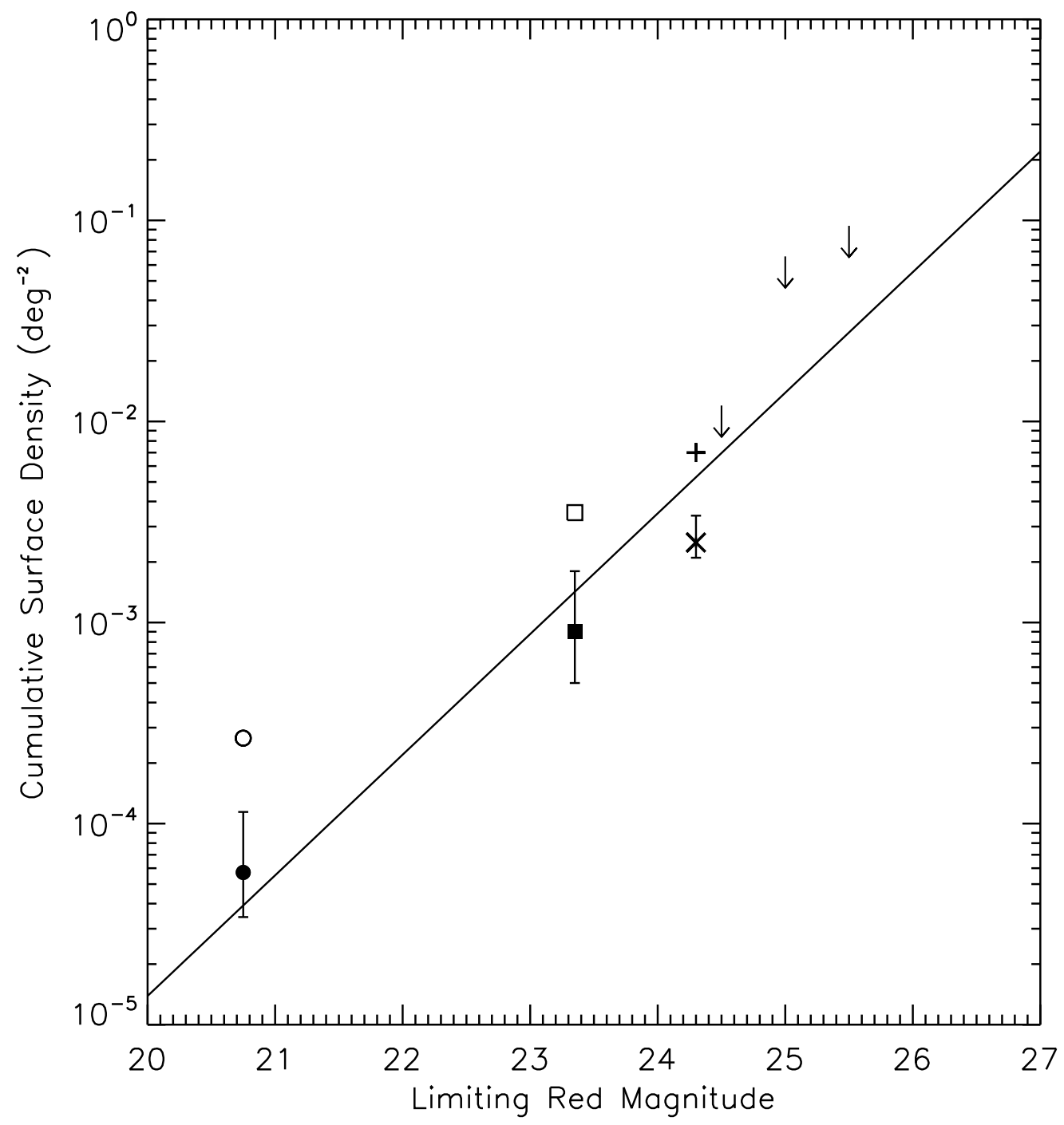

Fig. 16. - The Cumulative Luminosity Function for Inner Oort Cloud Objects in the r-band. Sedna is shown by the filled circle while 2012 VP113 is shown by the filled square. For null detections, the three sigma upper limits are shown with arrows. The solid line is the Kuiper Belt luminosity function shifted downwards to match the two known IOC detections. The X shows the two extreme detached trans-Neptunian objects (2014 SR349 and 2013 FT28) along with the 2012 VP113 detection from our survey. This result is based on uniform sky coverage of IOC objects. If they are clustered in longitude of perihelion between about 2 and 7 hours in right ascension, their cumulative surface densities would hold only in that region of sky and be increased by over a factor of four as shown by the open symbols. The plus shows just 2014 SR349 and 2012 VP113 assuming clustered longitude of perihelion as 2013 FT28 would not be found in this region. 


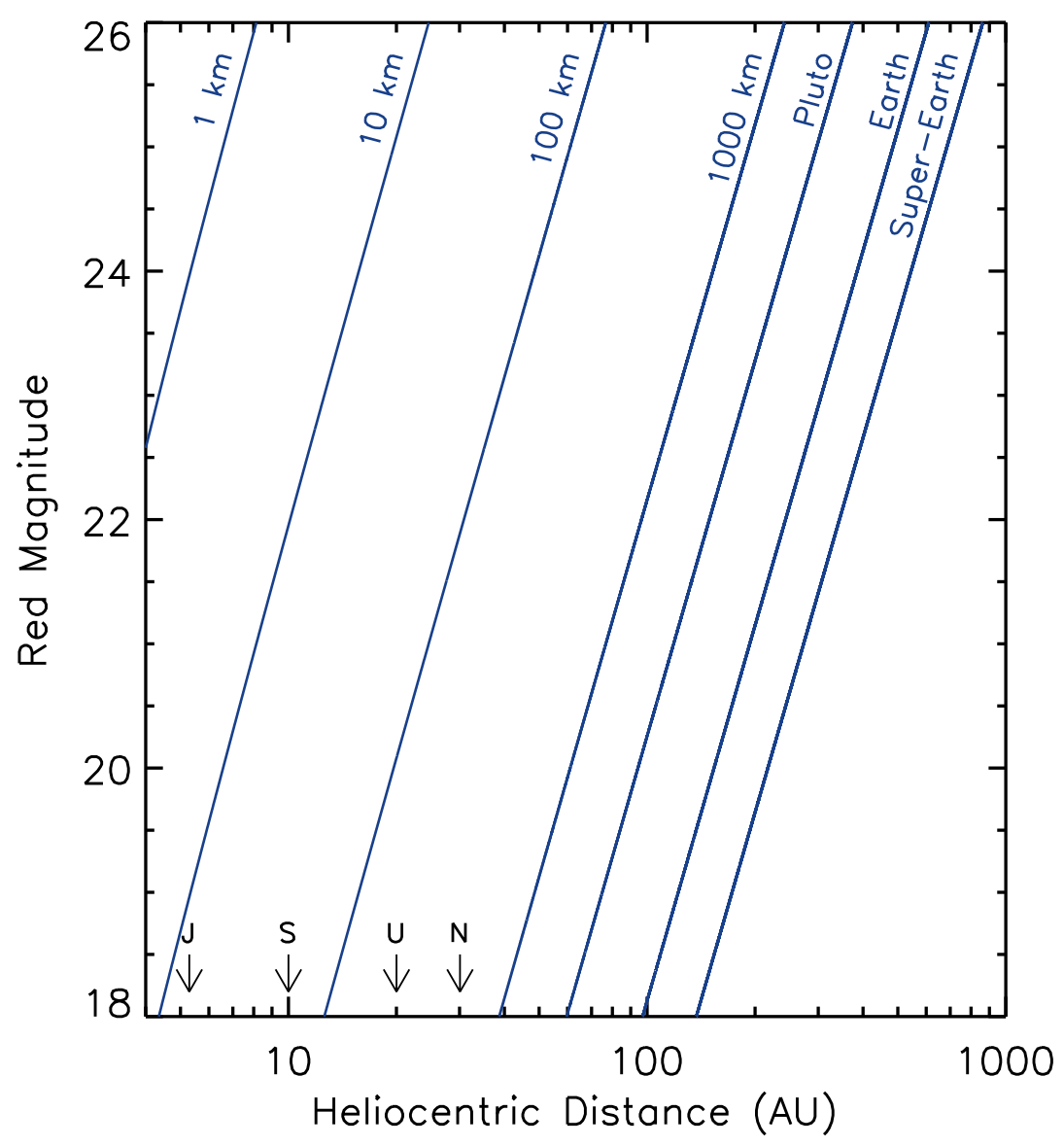

Fig. 17.- The r-band magnitude of different sized objects and heliocentric distances assuming a moderate albedo of 0.20. A super-Earth of about 10 Earth masses would have about twice the radius of Earth. The biggest telescopes with wide-field imagers can efficiently survey to just below 26th magnitude. Thus a Super-Earth beyond several hundred AU would likely be fainter than any current wide-field survey capability. 